\title{
ACCESS TO ANTIRETROVIRAL TREATMENT IN SOUTH AFRICA, 2004 - 2011
}

Leigh F Johnson, $P h D$

Centre for Infectious Disease Epidemiology and Research, University of Cape Town

Background. South Africa's National Strategic Plan (NSP) for 2007 - 2011 aimed to achieve new antiretroviral treatment (ART) enrolment numbers equal to $80 \%$ of the number of newly eligible individuals in each year, by 2011.

Objectives. To estimate ART coverage in South Africa and assess whether NSP targets have been met.

Methods. ART data were collected from public and private providers of ART. Estimates of HIV incidence rates were obtained from independent demographic projection models. Adult ART data and incidence estimates were entered into a separate model that estimated rates of progression through CD4 stages, and the model was fitted to South African CD4 data and HIV prevalence data.

Results. By the middle of 2011, the number of patients receiving ART in South Africa had increased to 1.79 million $(95 \% \mathrm{Cl} 1.65-1.93$ million). Adult ART coverage, at the previous ART eligibility criterion of CD4<200/ $\mu$ l, was $79 \%$ (95\% Cl $70-85 \%$ ), but reduced to $52 \%(95 \% \mathrm{Cl} 46-57 \%)$ when assessed according to the new South African ART eligibility criteria (CD4<350/ $\mu$ l). The number of adults starting ART in 2010/11 was 1.56 times ( $95 \% \mathrm{Cl} 1.08$ - 1.97) the number of adults who became ART-eligible in 2010/11, well in excess of the $80 \%$ target. However, this ratio was substantially higher in women $(1.96,95 \% \mathrm{Cl} 1.33-2.51)$ than in men $(1.23,95 \% \mathrm{Cl} 0.83-$ $1.58)$ and children $(1.13,95 \% \mathrm{Cl} 0.74-1.48)$.

Conclusion. South Africa has exceeded the ART targets in its 2007 - 2011 NSP, but men and children appear to be accessing ART at a lower rate than women.

Antiretroviral treatment (ART) is a powerful tool for reducing both AIDS mortality,2 and HIV transmission. ${ }^{3}$ The monitoring of access to ART is therefore critical to the evaluation of the impact of HIV treatment and prevention programmes. Previous monitoring exercises have shown that, since the announcement of a comprehensive care, management and treatment programme by the South African Department of Health in late 2003, access to ART in South Africa has increased dramatically. ${ }^{4,5}$ These assessments suggested that South Africa was on track to meet the targets laid out in the 2007 - 2011 National Strategic Plan (NSP) for HIV/AIDS and Sexually Transmitted Infections, which aimed to achieve new ART enrolment numbers equal to $80 \%$ of the number of newly eligible individuals in each year, by $2011 .{ }^{6}$ However, there has not as yet been any formal assessment of whether this target has been met.

The monitoring of access to ART in South Africa is challenging for several reasons. The interpretation of public sector statistics is complicated by changes in reporting practices in late 2009, with most provinces switching from reporting numbers of patients cumulatively started on ART to numbers of patients currently on ART. Statistics from disease management programmes and programmes run by non-governmental organizations (NGOs) have not been routinely collected and reported. In addition, there is generally a lack of information on the age and sex of patients. This is particularly problematic in view of concerns that ART initiation rates may be lower among men than women. ${ }^{7-9}$
The estimation of ART coverage is also hampered by uncertainty regarding the 'treatment need', the denominator in the coverage calculation. Mathematical models have been used to estimate numbers of HIV-positive individuals with CD4 counts below different thresholds, but there is substantial uncertainty surrounding the rates of $\mathrm{CD} 4$ decline that are assumed in these models, and there is also growing recognition that these rates of $\mathrm{CD} 4$ decline may differ between populations. ${ }^{10}$ There is also concern that cross-sectional measures of ART coverage may fail to give a sense of recent programme performance, which is better reflected in the ratio of the number of patients starting ART in a year to the number of individuals becoming eligible for ART in the same year." ${ }^{11}$ The latter measure has the advantage of being consistent with the way in which the South African NSP targets are expressed, and is also less sensitive to model assumptions about rates of CD4 decline and ART eligibility criteria. ${ }^{11}$

The objective of this paper is to assess recent changes in access to ART in South Africa, and to evaluate the extent to which the 2007 - 2011 NSP treatment targets have been met. This study also aims to improve on previous work ${ }^{4}$ by including more recent programme statistics, by using locally relevant CD4 data in the estimation of the treatment need, by including $95 \%$ confidence intervals ( $\mathrm{Cls}$ ) in coverage estimates, and by estimating coverage separately for men, women and children. 


\section{METHODS \\ ART PROGRAMME STATISTICS}

Public sector ART programme statistics to the end of June 2011 were obtained from the South African Department of Health, and were adjusted to achieve consistency of definition (cumulative/ current), using a previously described formula ${ }^{4}$ for each province. Unpublished data on the sex ratio of adult patients enrolled in public ART programmes in four provinces, collected up to March 2009 , were used to estimate the sex ratio of adults starting ART in the public sector.

Private sector data and data from NGOs were obtained through surveys conducted every two years, since mid-2006. ${ }^{12}$ Linear interpolation and extrapolation was used to estimate numbers for programmes with missing data and for years in which no survey was conducted. Estimates of the proportion of private sector patients who were men, women and children were obtained from submissions by medical schemes to the Risk Equalization Fund up to March 2008, and the geographical distribution of private sector patients was estimated from early private sector statistics. ${ }^{13}$ Detailed data collected from NGO programmes in the 2008 survey were used to determine the profile of NGO patients by age, sex and province.

\section{MATHEMATICAL MODEL}

To estimate the numbers of adults needing ART, a mathematical model was developed to simulate the growth of the South African population over time, the incidence of HIV and the decline in CD4 counts in HIV-positive adults. The model stratifies the population by age and sex, and projects the change in population in one-year intervals, starting in the middle of 1985 . Assumptions regarding the age- and sex-specific population profile, non-HIV mortality, fertility, migration and HIV incidence are based on the ASSA2008 AIDS and Demographic model. ${ }^{14}$ Once infected, individuals are assumed to progress through a four-stage model of CD4 decline, in the absence of ART (Fig. 1). Individuals are assumed to experience AIDS mortality in the CD4 $200-349 / \mu$ l category at a fraction $\theta$ of the AIDS mortality rate in the $C D 4<200 / \mu$ l category, if untreated. Up to mid-2009, adults of sex $g$ are assumed to start ART only once their CD4 count has dropped below 200/ $\mu$, at a rate of $r(t)$ per annum in year $t$. Between mid-2009 and mid-2011, the model also allows individuals to start ART in the CD4 200 - 349 category if they develop tuberculosis or become pregnant, following the change in South African ART guidelines in early $2010 .{ }^{15}$ The $r_{g}(t)$ rates in each year are calculated from the ART programme statistics (further detail is provided in the online appendix).

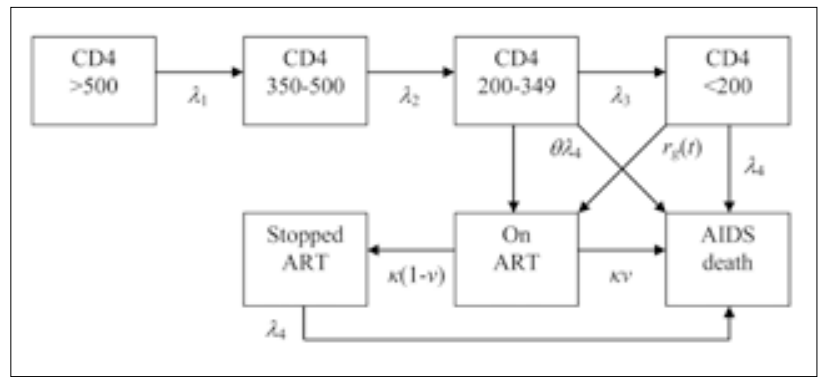

Fig. 1. Multi-state model of decline in CD4 count and ART initiation by HIV-infected adults. All states are stratified by age and sex, and all HIVinfected adults are assumed to experience age-specific mortality unrelated to HIV (not shown).

Adults who start ART are assumed to be lost to the ART programme with probability $\kappa_{0}$ during the first 6 months after starting ART, and with probability $\kappa_{1}$ for each year after the first 6 months. This does not include individuals who temporarily interrupt ART. Of those leaving the ART programme permanently, a proportion $v$ are assumed to leave the programme owing to HIV-related mortality, and the remaining proportion $(1-v)$ are assumed to stop taking their drugs, after which their mortality risk is assumed to be the

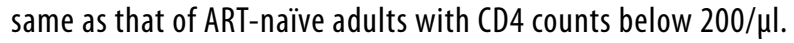

Estimates of annual numbers of new paediatric HIV infections were obtained from a separate model of paediatric HIV in South Africa. ${ }^{16}$ Since paediatric ART guidelines recommend ART initiation in all HIV-infected children aged $<12$ months, regardless of their immunological or clinical status, ${ }^{17}$ the annual number of new paediatric HIV infections is used to approximate the annual number of children newly eligible for ART (the denominator in the ART enrolment ratio).

\section{CALIBRATION AND UNCERTAINTY ANALYSIS}

The parameters determining the rates of CD4 decline, HIV-related mortality and ART discontinuation are estimated by fitting the model to HIV prevalence data from the 2005 and 2008 Human Sciences Research Council (HSRC) household surveys, ${ }^{18,19}$ and to CD4 data from HIV-positive adults in three South African surveys, ${ }^{20-22}$ using a Bayesian melding procedure. ${ }^{23,24}$ A detailed explanation is provided in the online appendix. Briefly, prior distributions are specified to represent uncertainty regarding the parameters of interest, including the range of plausible values for the average time to starting ART after becoming eligible $\left(1 / r_{g}(t)\right)$. Prior distributions are also specified to represent uncertainty regarding the accuracy of the reported ART programme statistics in each year. This uncertainty and the uncertainty regarding ART attrition rates affect the model ART enrolment inputs. A likelihood function is specified to represent how well the model fits the CD4 data and HIV prevalence data, for a given set of parameter values. The posterior distribution, representing the parameter combinations from the prior distributions that have the highest likelihood values, is then simulated by Sampling Importance Resampling. ${ }^{25}$

\section{RESULTS}

The posterior estimates of the model parameters are summarised in Table 1, and posterior estimates of numbers of patients receiving ART are summarised in Table 2. Over the period mid-2004 to mid2011, the total number of patients receiving ART in South Africa increased from $47500(95 \%$ Cl $42900-51800)$ to 1.79 million (95\% Cl 1.65 - 1.93 million). Of the latter, $85 \%$ were receiving ART through the public health sector, $11 \%$ were receiving ART through disease management programmes in the private sector, and the remaining $4 \%$ were receiving ART through community treatment programmes run by NGOs. The majority (61\%) of patients were women aged 15 or older, men accounted for $31 \%$ of patients, and children under the age of 15 comprised the remaining $8 \%$ of patients. KwaZulu-Natal and Gauteng were the two provinces with the largest numbers of patients, together accounting for $56 \%$ of all patients receiving ART.

Changes over time in numbers of treated and untreated adults in different CD4 stages are shown in Fig. 2. As at mid-2011, untreated HIV-positive adults included 58000 (95\% Cl $13000-147000)$ individuals who had stopped ART, $385000(95 \% \mathrm{Cl} 247000-634$ 000) ART-naive adults with $C D 4<200 / \mu \mathrm{l}, 1.06$ million $(95 \% \mathrm{Cl} 0.88$ - 1.29 million) with CD4 counts of $200-349 / \mu l, 0.74$ million ( $95 \%$ (I $0.61-0.91$ million) with CD4 counts of $350-500 / \mu l$, and 0.94 million ( $95 \%$ Cl $0.77-1.16$ million) with CD4 counts $>500 / \mu l$. The 


\section{TABLE 1. POSTERIOR ESTIMATES OF MODEL PARAMETERS}

\begin{tabular}{|c|c|c|}
\hline & Symbol & Mean $(95 \% \mathrm{Cl})$ \\
\hline \multicolumn{3}{|l|}{ Parameters for untreated adults } \\
\hline Annual rate of progression from CD4 $>500$ to $350-500$ & $\lambda_{1}$ & $0.34(0.28-0.39)$ \\
\hline Annual rate of progression from CD4 350 - 500 to $200-349$ & $\lambda_{2}$ & $0.48(0.40-0.58)$ \\
\hline Annual rate of progression from CD4 $200-349$ to $<200$ & $\lambda_{3}$ & $0.32(0.25-0.39)$ \\
\hline Annual rate of HIV mortality if CD4 $<200$ & $\lambda_{4}$ & $0.21(0.16-0.27)$ \\
\hline Ratio of HIV mortality at CD4 $200-349$ to HIV mortality at CD4 $<200$ & $\theta$ & $0.13(0.05-0.24)$ \\
\hline \multicolumn{3}{|l|}{ Parameters for treated adults } \\
\hline Probability of permanent loss to care in first 6 months after ART start & $\kappa_{0}$ & $0.078(0.028-0.141)$ \\
\hline Annual probability of permanent loss to care after first 6 months of ART & $k_{1}$ & $0.048(0.018-0.087)$ \\
\hline Proportion of permanent loss to care that is due to death & v & $0.74(0.53-0.92)$ \\
\hline
\end{tabular}

\begin{tabular}{|c|c|c|c|c|c|c|c|c|}
\hline & 2004 & 2005 & 2006 & 2007 & 2008 & 2009 & 2010 & 2011 \\
\hline \multicolumn{9}{|l|}{ Currently on ART* } \\
\hline Total & 47500 & 110900 & 235000 & 382000 & 588000 & 912000 & 1287000 & 1793000 \\
\hline \multicolumn{9}{|l|}{ By sex/age } \\
\hline Men & 17700 & 37500 & 75000 & 120000 & 183000 & 283000 & 396000 & 551000 \\
\hline Women & 25600 & 63600 & 138000 & 228000 & 354000 & 553000 & 777000 & 1090000 \\
\hline Children $(<15)$ & 4200 & 9800 & 22000 & 35000 & 51000 & 76000 & 113000 & 152000 \\
\hline \multicolumn{9}{|l|}{ By provider } \\
\hline Public sector & 9600 & 60600 & 163000 & 290000 & 470000 & 748000 & 1073000 & 1525000 \\
\hline Private sector & 34100 & 43800 & 57000 & 68000 & 86000 & 117000 & 154000 & 190000 \\
\hline NGO programmes & 3900 & 6400 & 15000 & 24000 & 32000 & 47000 & 60000 & 78000 \\
\hline \multicolumn{9}{|l|}{ By province } \\
\hline Eastern Cape & 5300 & 12600 & 26000 & 43000 & 65000 & 98000 & 137000 & 187000 \\
\hline Free State & 2200 & 4900 & 10000 & 18000 & 29000 & 47000 & 66000 & 91000 \\
\hline Gauteng & 13800 & 30800 & 62000 & 95000 & 145000 & 219000 & 280000 & 439000 \\
\hline KwaZulu-Natal & 12800 & 30300 & 67000 & 110000 & 174000 & 282000 & 409000 & 558000 \\
\hline Limpopo & 2000 & 4800 & 12000 & 21000 & 36000 & 60000 & 101000 & 124000 \\
\hline Mpumalanga & 3300 & 5800 & 12000 & 24000 & 38000 & 61000 & 96000 & 142000 \\
\hline Northern Cape & 400 & 1500 & 3000 & 7000 & 9000 & 13000 & 16000 & 19000 \\
\hline North West & 2700 & 8800 & 21000 & 34000 & 48000 & 70000 & 96000 & 126000 \\
\hline Western Cape & 5000 & 11400 & 21000 & 31000 & 45000 & 64000 & 85000 & 107000 \\
\hline \multicolumn{9}{|l|}{ Started ART last year ${ }^{\dagger}$} \\
\hline Men & 8400 & 22400 & 43000 & 52000 & 75000 & 118000 & 138000 & 189000 \\
\hline Women & 13700 & 42600 & 84000 & 104000 & 149000 & 235000 & 273000 & 380000 \\
\hline Children $(<15)$ & 2700 & 6400 & 13000 & 15000 & 20000 & 29000 & 45000 & 48000 \\
\hline Total & 24800 & 71300 & 140000 & 172000 & 243000 & 382000 & 456000 & 617000 \\
\hline \multicolumn{9}{|c|}{$\begin{array}{l}\text { All numbers are rounded to the nearest } 1000 \text { (except in the case of } 2004 \text { and } 2005 \text { totals, which are rounded to the nearest } 100) \text {. Due to rounding, some rows may not sum to the total. All estimates } \\
\text { posterior averages ( } 95 \% \text { confidence intervals not shown). } \\
\text { *Totals reflect numbers at the middle of each year. } \\
{ }^{\dagger} \text { Totals reflect ART enrolment over the } 12 \text { months up to the middle of the year. }\end{array}$} \\
\hline
\end{tabular}

total unmet need in the middle of 2011 (ART-naïve adults with CD4 $<350 / \mu$ l plus all adults who had stopped ART) was 1.50 million (95\% Cl $1.24-1.84$ million), which is $32 \%$ lower than the total unmet need four years previously. Estimates of adult ART coverage and ART enrolment ratios are shown in Fig. 3. Using previous CD4 thresholds for defining ART eligibility $(C D 4<200 / \mu l)$, the fraction of adults eligible to receive ART who were actually on ART increased from $5.1 \%$ (95\% Cl $4.2-6.1 \%)$ in the middle of 2004 to $79 \%$ (95\% (I $70-85 \%$ ) by the middle of 2011. However, using the new CD4 thresholds for defining ART eligibility (CD4 $<350 / \mu l)$, adult ART coverage by the middle of 2011 was $52 \%$ (95\% Cl $46-57 \%)$.

As noted previously, ${ }^{11}$ ART enrolment ratios are similar when using different CD4 thresholds to define ART eligibility. For example, 
over the period from mid-2010 to mid-2011, the ratio of the number of adults starting ART to the number of adults whose CD4 counts fell below the CD4 threshold was 1.64 (95\% Cl 1.11 - 2.10) when the CD4 threshold was 200 , and 1.56 (95\% Cl 1.08 - 1.97) when the CD4 threshold was 350 . Both ratios are roughly double the target of $80 \%$ set in the 2007 - 2011 NSP, and indicate substantial progress in removing the 'backlog' of unmet need that accumulated in previous years.

Estimates of ART access are presented separately for men, women and children in Fig. 4. Using the CD4 threshold of 350/ $\mu$ las the criterion for ART eligibility, the fraction of ART-eligible women who were receiving ART by the middle of 2011 (60\%, 95\% CI 53 - $65 \%$ ) was significantly higher than the fraction of ART-eligible men who were on treatment $(41 \%, 95 \%$ CI $36-46 \%)$. A similar difference in magnitude is seen in the ART enrolment ratio over the period mid-2010 to mid-2011: using the same ART eligibility criterion of $\mathrm{CD} 4<350 / \mu \mathrm{l}$, the enrolment ratio was $1.96(95 \% \mathrm{Cl}$ $1.33-2.51)$ in women and $1.23(95 \% \mathrm{Cl} 0.83-1.58)$ in men. Over the same period, the ratio of the number of children starting ART to the number of new infections in children was $1.13(95 \% \mathrm{Cl}$ $0.74-1.48)$. In most previous years, this ratio was below both the male ART enrolment ratio and the female ART enrolment ratio.

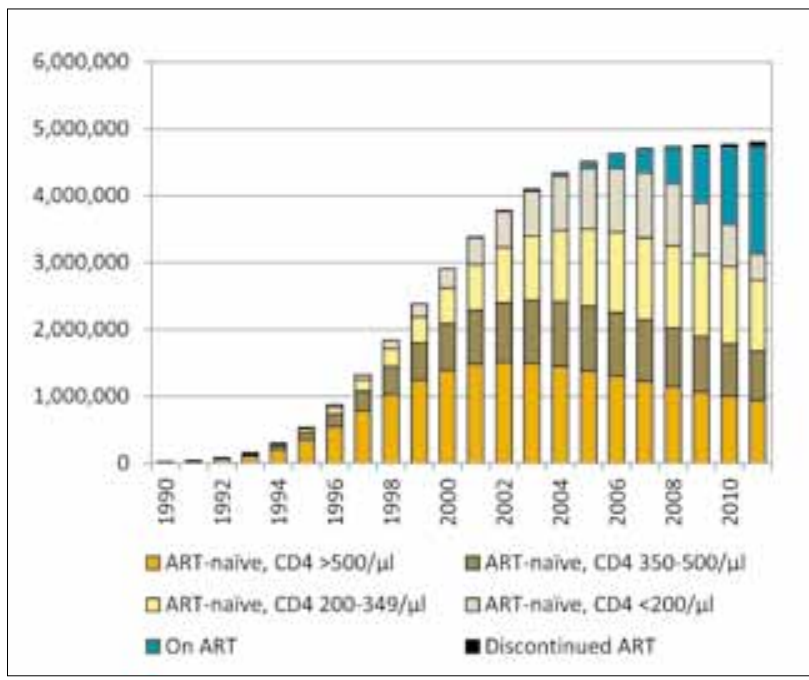

Fig. 2. Numbers of HIV-positive adults, by CD4 count and ART status. Numbers exclude paediatric HIV infections. Bars represent posterior means (95\% confidence intervals not shown).

\section{DISCUSSION}

South Africa has made impressive progress in the rollout of ART since the start of the public sector ART programme in 2004. The number of patients who started ART in 2010/2011 was well in excess of the number of individuals who became eligible to receive ART over the same period, exceeding the targets set in the 2007 - 2011 NSP. The unmet need for ART was also reduced by $32 \%$ between 2007 and 2011. According to the ART initiation criteria that were in place at the time, adult treatment coverage by mid2011 was close to $80 \%$.

However, there appear to be substantial differences between men, women and children in the rate of ART initiation. The low rate of ART initiation in men relative to women may be a reflection of gender differences in health-seeking behaviour and perceptions that men who seek care are 'weak'. ${ }^{9}$ Alternatively, the high rate of ART initiation in women may be due to higher rates of HIV diagnosis through antenatal screening. The relatively low rates of ART initiation in children are probably attributable to the lower rates of HIV testing in children and the greater complexity of paediatric ART relative to adult ART ${ }^{26}$ However, it is difficult to compare adult and paediatric measures of ART access meaningfully because the course of HIV infection is so different in children, with many HIVinfected infants dying in the first few months of life before there is an opportunity for testing.

This analysis extends previous work ${ }^{4}$ by including assessment of uncertainty and by incorporating several new data sources. The 95\% Cls that have been estimated reflect uncertainty regarding rates of CD4 decline, rates of mortality and rates of ART retention, and also reflect uncertainty regarding the accuracy of reported ART programme statistics. However, the Cls do not reflect the uncertainty regarding the HIV incidence rates that have been estimated from the ASSA2008 model, and this may lead to some exaggeration of precision. Cls around the ART enrolment ratios are considerably wider in 2009/10 and 2010/11 than in previous years, owing to the change in the way that the Department of Health has reported public sector ART programme statistics.

Various attempts were made to validate the reported ART programme statistics using data from external sources, with limited success. Lamivudine sales figures from Aspen Pharmacare, which until recently supplied $80 \%$ of lamivudine in the public sector, were used to obtain crude estimates of numbers of public sector patients on treatment in each quarter. These estimates were (a) ART coverage

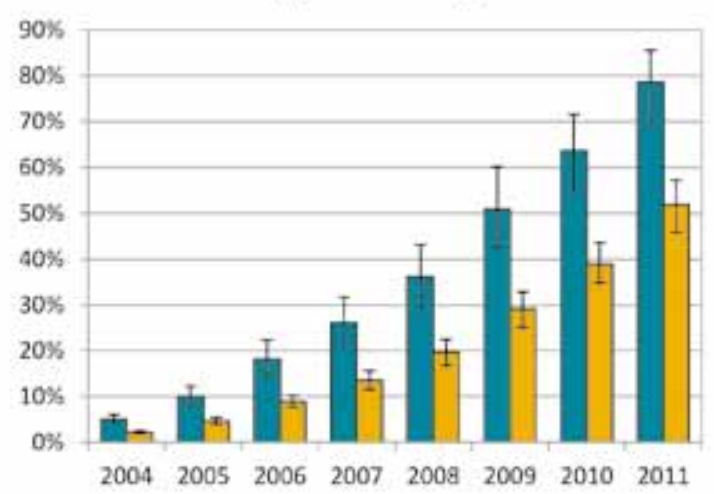

(b) ART enrolment ratio

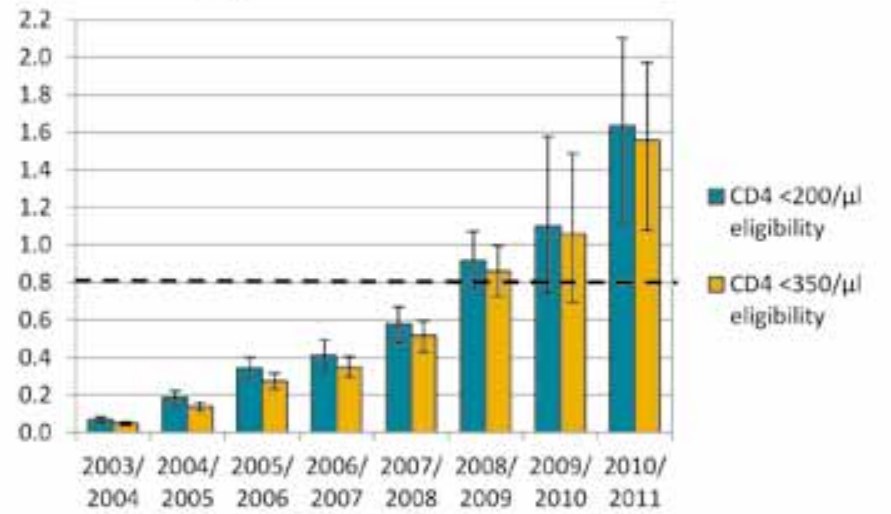

Fig. 3. Adult ART access. Bars represent posterior means and error bars represent $95 \%$ confidence intervals. Dashed line in panel (b) represents 2007 2011 National Strategic Plan target. 
(a) ART coverage at CD4<350

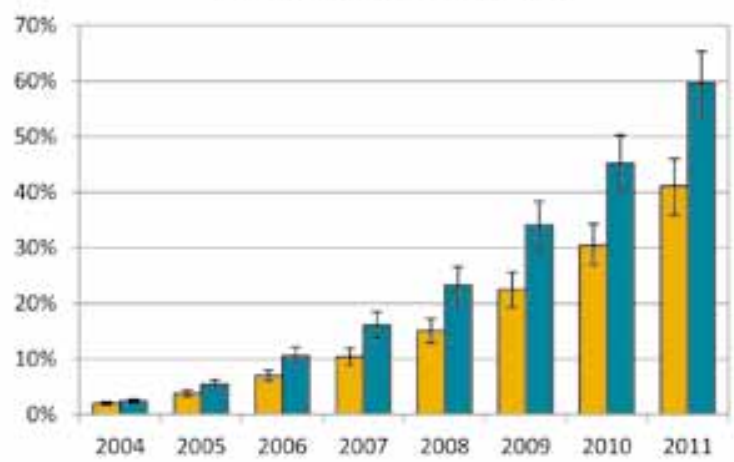

(b) ART enrolment ratio

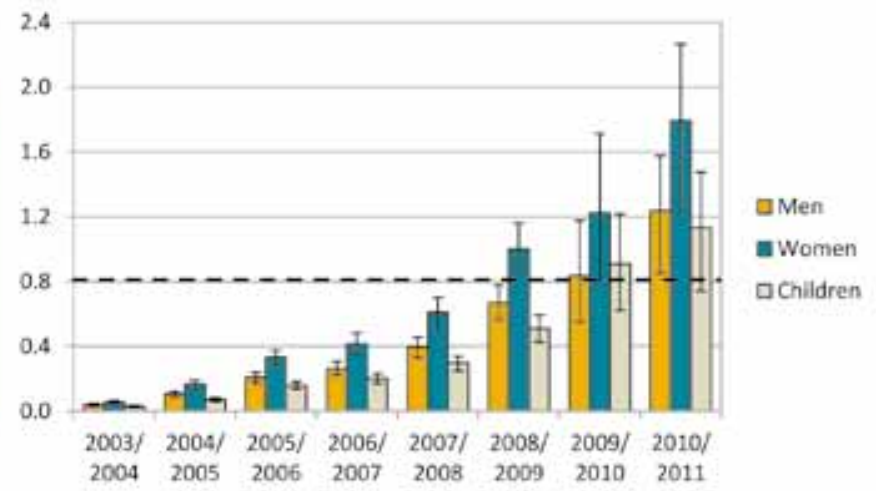

Fig. 4. Age and sex differences in ART access. Bars represent posterior means and error bars represent $95 \%$ confidence intervals. Dashed line in panel (b) represents 2007 - 2011 National Strategic Plan Target.

not significantly different from the model estimates in Table 2 up to the end of 2008, and from October 2009 to March 2010, but were substantially lower than the model estimates from January to September of 2009. Numbers of viral load tests performed by the National Health Laboratory Service for public sector clinics were also used to obtain theoretical estimates of numbers of patients receiving $A R T$, on the assumption that patients went for viral load testing twice per annum on average. The resulting estimates were slightly higher than the corresponding model estimates up to 2008 , but were $18 \%$ lower than the model estimates in 2009 . Finally, the model estimate of the fraction of the 15 - 49-yearold population on ART in the middle of 2008 was compared with the corresponding proportion estimated in the $2008 \mathrm{HSRC}$ national household survey, ${ }^{27}$ based on testing for the presence of antiretroviral drugs in blood samples: the model estimate of $1.8 \%$ (95\% Cl $1.6-2.0 \%)$ was found to be significantly lower than that measured in the survey (3.0\%). External data sources therefore do not provide a clear and consistent assessment of the plausibility of the model estimates derived from reported ART programme statistics.

Although attempts were made to produce estimates of ART coverage for each province, it was not possible to produce plausible results for two provinces (Gauteng and Western (ape) because the estimated numbers of patients starting ART in recent years exceeded the estimated numbers of patients eligible to receive ART, in both of these provinces. This could possibly be due to individuals with advanced HIV migrating to urban areas because of the perceived superiority of health services in the major urban centres of Gauteng and Western Cape. The model assumes migration to be independent of HIV status, and may therefore under-estimate the number of HIV-infected ART-eligible individuals who migrate into these provinces. Alternatively, the problems experienced in producing plausible results for Gauteng and Western Cape may be due to assumed HIV incidence rates in these provinces being too low, or reported numbers of ART patients in these provinces being exaggerated.

Many challenges exist, both in achieving future ART rollout targets and in monitoring future progress towards meeting these targets. The new NSP for the 2012 - 2016 period $^{28}$ proposes targets that are much more ambitious than those in the previous NSP: the ART enrolment target in 2016 is $80 \%$ of the new ART need in that year plus $80 \%$ of the unmet need from previous years. High levels of HIV testing and counselling, as well as expansion of capacity to deliver ART, will be required to meet these targets. The new NSP for the 2012 - 2016 period proposes several measures to strengthen the monitoring and evaluation of South Africa's ART programme, including the introduction of a single patient identifier in the health sector and a single registry at the primary care level. It is hoped that these measures will lead to greater precision in the estimation of ART coverage in future, as well as a deeper understanding of the factors determining access to care and retention in care.

Appearing only in the online version of this article is an appendix that provides further detail regarding the method used to model adult ART initiation. It also includes a detailed explanation of the Bayesian melding procedure: the prior distributions and the data sources on which they are based, the method used to define the likelihood function and the method used to simulate the posterior distribution.

\section{Acknowledgements}

I am grateful to the many disease management programmes and NGOs that shared data, as well as the National Health Laboratory Service and Aspen Pharmacare for providing data for validation purposes.

\section{REFERENCES}

1. Floyd $S$, Molesworth A, Dube A, et al. Population-level reduction in adult mortality after extension of free anti-retroviral therapy provision into rural areas in northern Malawi. PLoS One 2010:5(10):e13499. [http://dx.doi.org/10.1371/journal.pone.0013499] [PMID:20976068].

2. Herbst AJ, Cooke GS, Bärnighausen T, KanyKany A, Tanser F, Newell ML. Adult mortality and antiretroviral treatment roll-out in rural KwaZulu-Natal, South Africa. Bull WHO 2009;87(10):75462. [http://dx.doi.org/10.2471/BLT.08.058982] [PMID:19876542].

3. Cohen MS, Chen YQ, McCauley M, et al. Prevention of HIV-1 infection with early antiretroviral therapy. N Engl J Med 2011;365(6):493-505. [http://dx.doi.org/10.1056/NEJMoa1105243] [PMID:21767103].

4. Adam MA, Johnson LF. Estimation of adult antiretroviral treatment coverage in South Africa. S Afr Med J 2009;99(9):661-667. [PMID:20073293].

5. Klausner JD, Serenata $\mathrm{C}, \mathrm{O}^{\prime} \mathrm{Bra} \mathrm{H}$, et al. Scale-up and continuation of antiretroviral therapy in South African treatment programs, 2005-2009. J Acquir Immun Defic Syndr 2011;56(3):292-295. [http:// dx.doi.org/10.1097/QAl.0b013e3182067d99] [PMID:21317587].

6. Department of Health. HIV and AIDS and STI Strategic Plan for South Africa, 2007-2011. http:// www.doh.gov.za/docs/strategicplan/2007/part1.pdf (accessed 6 January 2012).

7. Cornell M, Mclntyre J, Myer L. Men and antiretroviral therapy in Africa: our blind spot. Trop Med Int Health 2011;16(7):828-829. [http://dx.doi.org/10.1111/j.1365-3156.2011.02767.x] [PMID:21418449].

8. Muula AS, Ngulube TJ, Siziya $S$, et al. Gender distribution of adult patients on highly active antiretroviral therapy (HAART) in Southern Africa: a systematic review. BMC Public Health 2007;7:63. [http://dx.doi.org/10.1186/1471-2458-7-63] [PMID:17459154].

9. Nattrass N. Gender and access to antiretroviral treatment in South Africa. Fem Econ 2008;14(4):19-36.

10. Williams BG, Korenromp EL, Gouws E, Schmid GP, Auvert B, Dye C. HIV infection, antiretroviral therapy, and CD4+ cell count distributions in African populations. J Infect Dis 2006;194(10):14501458. [http://dx.doi.org/10.1086/508206] [PMID:17054076].

11. Johnson LF, Boulle A. How should access to antiretroviral treatment be measured? Bull WHO 2011;89:157-160. [http://dx.doi.org/10.2471/BLT.10.080911] [PMID:21346928].

12. Johnson LF, McLeod HD. Steady growth in antiretroviral treatment provision by disease management and community treatment programmes. S Afr Med J 2007;97(5):358-359. [PMID:17599217].

13. Regensberg LD, Hislop MS. A report back on more than four years of HIV/AIDS disease management in southern Africa. South Afr J HIV Med 2003;4(1):7-10.

14. Actuarial Society of South Africa. ASSA2008 AIDS and Demographic Model. 2011. http://aids. 
actuarialsociety.org.za (accessed 5 April 2011)

15. Department of Health. Clinical Guidelines for the Management of HIV and AIDS in Adults and Adolescents. 2010. http://www.doh.gov.za/docs/facts-f.html (accessed 30 July 2010).

16. Johnson LF, Stinson K, Newell ML, et al. The contribution of maternal HIV seroconversion during late pregnancy and breastfeeding to mother-to-child transmission of HIV. J Acquir Immun Defic Syndr 2012 (in press). [http://dx.doi.org/10.1097/QAl.0b013e3182432f27] [PMID:22193774].

17. Department of Health. Guidelines for the Management of HIV in Children. 2010. http://www.doh. gov.za/docs/index html (accessed 6 March 2011).

18. Shisana 0, Rehle T, Simbayi LC, et al. South African National HIV Prevalence, HIV Incidence, Behaviours and Communication Survey, 2005. Cape Town: HSRC Press, 2005. http://www.hsrcpress. ac.za (accessed 1 December 2005).

19. Shisana 0, Rehle T, Simbayi LC, et al. South African national HIV Prevalence, Incidence, Behaviour and Communication Survey, 2008: A Turning Tide among Teenagers? Cape Town: Human Sciences Research Council, 2009. http://www.hsrcpress.ac.za (accessed 9 June 2009).

20. Auvert B, Males S, Puren A, Taljaard D, Carael M, Williams B. Can highly active antiretroviral therapy reduce the spread of HIV? A study in a township of South Africa. J Acquir Immun Defic Synd 2004:36(1):613-21. [http://dx doi org/10.1097/00126334-200405010-00010] [PMID:15097305].

21. Rehle TM, Shisana 0. Estimates of eligibility for antiretroviral treatment (ART) and projected
ART impact on AIDS mortality among South African educators. SAHARA J 2005;2(3):304-310. [PMID:17600972].

22. Connelly D, Veriava $Y$, Roberts $S$, et al. Prevalence of HIV infection and median $C D 4$ counts among health care workers in South Africa. S Afr Med J 2007;97(2):115-120. [PMID:17404672].

23. Alkema L, Raftery AE, Clark SJ. Probabilistic projections of HIV prevalence using Bayesian melding. Ann Appl Stat 2007;1(1):229-248. [http://dx.doi.org/10.1214/07-A0AS111]

24. Poole D, Raftery AE. Inference for deterministic simulation models: The Bayesian melding approach. J Am Stat Assoc 2000;95(452):1244-1255. [http://dx.doi.org/10.2307/2669764].

25. Smith AFM, Gelfand AE. Bayesian statistics without tears - a sampling resampling perspective. Am Stat 1992;46(2):84-88. [http://dx.doi.org/10.2307/2684170]

26. Meyers $T$, Moultrie H, Naidoo K, Cotton M, Eley B, Sherman G. Challenges to pediatric HIV care and treatment in South Africa. J Infect Dis 2007;196(Suppl 3):S474-481. [http://dx.doi. org/10.1086/521116] [PMID:18181697].

27. Rehle TM, Hallett TB, Shisana 0, et al. A decline in new HIV infections in South Africa: estimating HIV incidence from three national HIV surveys in 2002, 2005 and 2008. PLoS One 2010;5(6):e11094. [http://dx.doi.org/10.1371/journal.pone.0011094] [PMID:20559425].

28. South African National AIDS Council. National Strategic Plan on HIV, STIs and TB, 2012-2016. 2011. http://www.doh.gov.za/docs/stratdocs/2011/summary_hiv_nsp.pdf (accessed 6 December 2011). 


\section{Appendix}

This appendix provides further detail regarding the method used to model adult ART initiation. It also includes a detailed explanation of the Bayesian melding procedure: the prior distributions and the data sources on which they are based, the method used to define the likelihood function and the method used to simulate the posterior distribution.

\section{Method used to model adult ART initiation}

The model inputs are the numbers of adults of sex $g$ starting ART in year $t, S_{g}(t)$. These are used to calculate $r_{g}(t)$, the rate of ART initiation in adults of sex $g$, who are ART-naïve and with CD4 counts $<200 / \mu 1$, during year $t$. Prior to the change in ART guidelines in 2009 , the rate $r_{g}(t)$ can be calculated in terms of $S_{g}(t)$, if we have the model estimates of the numbers of individuals who are ART-eligible at the start of year $t, E_{g}(t)$, as well as the numbers who become eligible over the course of year $t, P_{g}(t)$, and the rate of mortality in untreated ARTeligible adults, $\lambda_{4}$. If it is assumed that the individuals who progress to CD $4<200 / \mu 1$ do so uniformly over the course of the year, it can be shown that

$$
\begin{aligned}
S_{g}(t)= & E_{g}(t) \frac{r_{g}(t)}{r_{g}(t)+\lambda_{4}}\left[1-\exp \left(-r_{g}(t)-\lambda_{4}\right)\right] \\
& +P_{g}(t) \frac{r_{g}(t)}{r_{g}(t)+\lambda_{4}}\left\{1-\frac{1}{r_{g}(t)+\lambda_{4}}\left[1-\exp \left(-r_{g}(t)-\lambda_{4}\right)\right]\right\}
\end{aligned}
$$

This formula is used to calculate the rate of ART initiation, $r_{g}(t)$, using Newton's method. Following the change in ART guidelines in 2009, a modified version of the above equation is used, to exclude individuals with CD4 counts of 200-349/ $\mu$ l who start ART when they have TB or are pregnant. If $B_{g}(x, t)$ is the model estimate of the number of adults aged $x$, of sex $g$, who have CD4 counts of 200-349/ $\mu$ at the start of year $t$, then the left hand side of the above equation changes to

$$
S_{g}(t)-\Omega(t) \sum_{x} B_{g}(x, t)\left(f_{g}(x, t)+u\right),
$$

where $\Omega(t)$ is the fraction of HIV-positive TB patients and pregnant women who start ART in year $t$, if their CD4 count is below $350 / \mu 1, f_{g}(x, t)$ is the fertility rate in year $t$ at age $x$, and $u$ is the TB incidence rate in HIV-positive individuals with CD4 counts of 200-349/ $\mu$. Equation (1) is therefore modified so that the left-hand side includes only those individuals who start ART with a CD4 count of $<200 / \mu 1$. A limitation of this formula is that it considers only the number of adults with CD4 200-349/ $\mu 1$ at the start of the year, and does not consider movements into and out of the CD4 200-349/ $\mu$ l state during the course of the year. However, since the movements into and out of the CD4 200-349/ $\mu$ l state offset one another to some extent, the change in $B_{g}(x, t)$ over a single year is likely to be modest, and the approximation is therefore reasonable.

The parameters in the above equation are estimated from various sources. The fertility rates by age and year are obtained from the ASSA2008 AIDS and Demographic model, ${ }^{1}$ and have 
been set to zero in the case of males. The annual TB incidence rate $u$, in adults with CD4 $200-349 / \mu 1$, is set to 0.043 , based on data from HIV patients in two provinces, Gauteng and Mpumlanga. ${ }^{2,3}$ The proportion of TB patients and pregnant women who are assumed to start ART, if their CD4 count is in the CD4 200-349 range, is set to 30\% in 2009/10 (since the change in guideline was only announced at the end of 2009 and formal guidelines were only published in early 2010) and to $75 \%$ in 2010/11. The latter assumption may be optimistic, as South African studies have generally found that the proportion of ART-eligible pregnant women who initiate ART before delivery is typically $30-60 \%,{ }^{4-7}$ and only one South African study has found the proportion to be as high as $75 \% .^{8}$

The same assumptions regarding fertility rates and TB incidence rates are used in allowing for ART initiation in the CD4 200-349/ $\mu$ l category.

\section{Prior distributions}

Prior distributions are specified for various model parameters to reflect the extent of uncertainty that exists a priori, before calibrating the model to CD4 data and HIV prevalence data. The sections that follow provide the justification for the prior distributions that have been chosen for each parameter. In general, gamma distributions have been chosen to represent uncertainty regarding parameters that can take on any positive value, beta distributions have been chosen to represent uncertainty regarding parameters that can take on any value between 0 and 1 , and normal distributions have been chosen to represent uncertainty regarding parameters that can take on any value. The distributions have therefore been chosen according to the range of values that they support, since the gamma, beta and normal distributions are defined on the ranges $[0, \infty),[0,1]$ and $(-\infty, \infty)$ respectively.

(a) Rates of CD4 decline in the absence of ART

The model that is used to describe progression to death in HIV-infected adults, in the absence of ART, is illustrated in Figure 1. All infected adults are assumed to have initial CD4 counts above 500 (based on the CD4 distributions observed in HIV-negative South Africans ${ }^{9,}{ }^{10}$ ), which decline over the course of HIV infection. The risk of AIDS mortality is assumed to begin after the CD4 count drops below 350 .

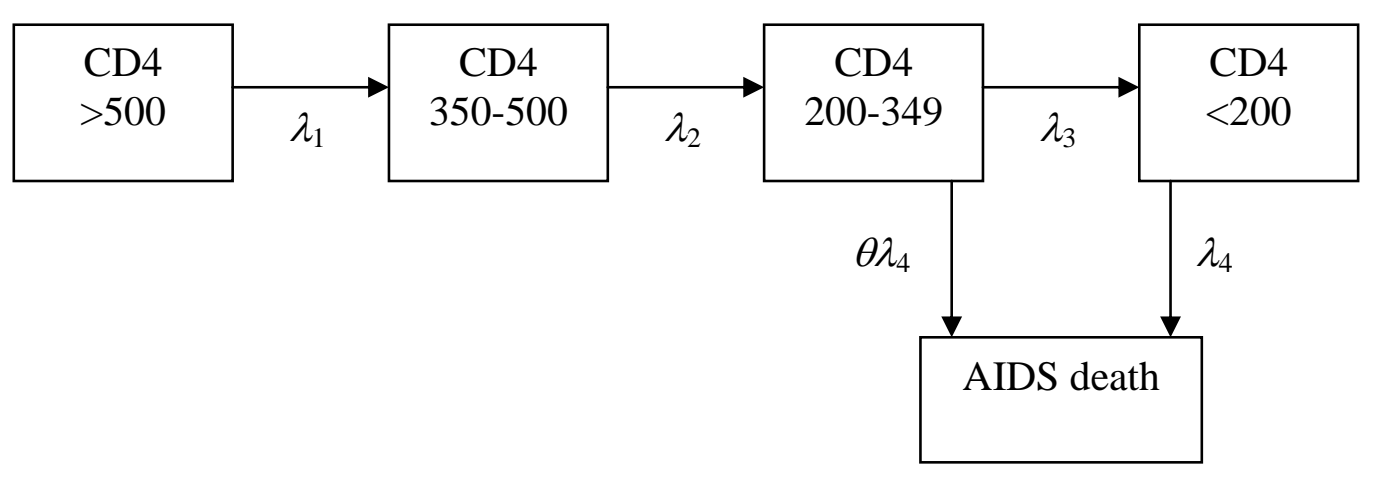

Figure 1: Multi-state model of decline in CD4 count in HIV-infected adults 
In setting the prior distributions on the rates of CD4 decline $\left(\lambda_{1}, \lambda_{2}\right.$, and $\left.\lambda_{3}\right)$, we review estimates from other studies that have attempted to estimate rates of CD4 decline in untreated HIV-positive individuals, prior to the availability of ART. Estimates obtained from three studies are summarized in Table 1 . All of these studies are based on cohorts in high income countries with HIV-1 subtype B epidemics, and the rates of CD4 decline estimated in these studies might therefore not be appropriate to South Africa. Estimates of $\lambda_{1}$ lie between 0.38 and 0.60 (average of 0.44 ), but these values are likely to be over-estimates of the rate of transition out of the CD4 >500 state. This is because in all three studies the CD4 >500 category was actually separated into three states (CD4 >900, CD4 700-900 and CD4 500699), and the transition intensities shown in Table 1 are the rates of transition from the CD4 500-699 state to the CD4 350-499 state. (Calculating the rate of transition out of the $>500$ category would mean increasing the denominator without changing the numerator.) Because of the likely over-statement, we set the prior distribution for the $\lambda_{1}$ parameter to be gamma with a mean of 0.3 and a standard deviation of 0.06 (so that the 97.5 percentile of the distribution is 0.43 , close to the average of the studies in Table 1 ).

Estimates of the $\lambda_{2}$ parameter lie between 0.30 and 0.51 (average value of 0.38 ). We choose a gamma distribution with a mean of 0.40 and a standard deviation of 0.08 to represent our prior uncertainty regarding this parameter. The 2.5 and 97.5 percentiles of this distribution are 0.26 and 0.58 respectively, an interval that includes all of the empirical estimates in Table 1. Finally, estimates of $\lambda_{3}$ lie between 0.26 and 0.49 . However, the estimate of Longini et al ${ }^{11}$ is likely to be an over-estimate because the model assumes that individuals can only develop AIDS-defining illnesses when their CD4 count is $<200$, so that individuals who develop AIDS with CD4 $>200$ are artificially "pushed through" into the CD4 $<200$ category. If the estimate of Longini et al is excluded, the average estimate of the $\lambda_{3}$ parameter is 0.36 . To represent the prior uncertainty regarding this parameter we therefore choose a gamma distribution with a mean of 0.35 and a standard deviation of 0.07 . The 2.5 and 97.5 percentiles of this distribution are 0.23 and 0.50 respectively.

Table 1: Estimated annual rates of CD4 decline in the absence of ART

\begin{tabular}{|c|c|c|c|c|}
\hline \multirow{4}{*}{ Study } & \multirow{4}{*}{ Population } & \multicolumn{3}{|c|}{ Rate of CD4 decline from } \\
\hline & & $>500$ to & $350-500$ to & $200-349$ \\
\hline & & $350-500$ & $200-349$ & to $<200$ \\
\hline & & $\left(\lambda_{1}\right)$ & $\left(\lambda_{2}\right)$ & $\left(\lambda_{3}\right)$ \\
\hline Satten \& Longini ${ }^{12^{*}}$ & $\begin{array}{l}\text { Men in San Francisco, } \\
1984-92\end{array}$ & 0.42 & 0.37 & 0.49 \\
\hline \multirow[t]{4}{*}{ Hendriks et $a l^{13^{*}}$} & Men in Amsterdam, 1985-90 & 0.38 & 0.34 & 0.34 \\
\hline & Men in Amsterdam, 1990-97 & 0.39 & 0.30 & 0.26 \\
\hline & Men in Vancouver, 1985-90 & 0.40 & 0.37 & 0.31 \\
\hline & Men in Vancouver, 1990-97 & 0.45 & 0.36 & 0.39 \\
\hline Longini et $a l^{11}$ & $\begin{array}{l}\text { USA army personnel, } \\
1985-90\end{array}$ & 0.60 & 0.51 & 0.49 \\
\hline Average & & 0.44 & 0.38 & 0.38 \\
\hline
\end{tabular}


(b) Rates of HIV mortality in the absence of ART

In setting the prior distributions to represent the uncertainty regarding the mortality rates in different CD4 categories, we review African studies of mortality in HIV-positive individuals not receiving ART. Table 2 summarizes the evidence from various African studies. In one study, ${ }^{14}$ mortality rates were reported both for patients receiving cotrimoxazole (CTX) and patients not receiving CTX, but only the former is included in Table 2, as the provision of CTX to HIV-positive patients has been the standard of care in South Africa for many years. In seven studies that estimated the annual mortality rate in patients with CD4 below $200 / \mu 1$, who were not receiving ART, estimates ranged from 0.14 to 0.39 (average value of 0.26 ). To represent the prior uncertainty regarding the $\lambda_{4}$ parameter we therefore choose a gamma prior with a mean of 0.25 and a standard deviation of 0.07 (this distribution has 2.5 and 97.5 percentiles of 0.13 and 0.40 respectively).

Estimates of the ratio of mortality in the CD4 200-349/ $\mu$ l category to that in the $<200 / \mu 1$ category $(\theta)$ range between 0.12 and 0.30 (average value of 0.22 ). However, these are likely to be over-estimates for two reasons. Firstly, because CD4 testing is not frequent, the CD4 count at the time of death is likely to be lower than the last measured CD4 count; this means that the rate of mortality in the CD4 200-349/ $\mu$ l category is likely to be over-estimated if it is assumed that the CD4 count at death is the same as the same as the last measured CD4 count. Secondly, CD4 measurements are highly variable, with recorded CD4 values fluctuating considerably around a notional 'true' value, even in the course of a single day. ${ }^{15-17}$ Since we are modelling only the 'true' value and not the fluctuations in recorded values, and since the mortality risk is likely to depend on the cumulative incidence of opportunistic infections over several days or weeks, the strength of association between the mortality risk and the true CD4 is likely to be understated by the observed association between the mortality risk and the measured CD4. A stronger association would imply a lower value of $\theta$. We therefore set the prior distribution for $\theta$ to be beta with a mean of 0.15 and a standard deviation of 0.05 (the 2.5 and 97.5 percentiles of this distribution are 0.07 and 0.26 respectively).

Table 2: Estimated rates of mortality in the absence of ART

\begin{tabular}{|c|c|c|c|c|c|}
\hline \multirow{2}{*}{ Study } & \multirow{2}{*}{ Population } & \multirow{2}{*}{$\begin{array}{l}\text { Frequency } \\
\text { of CD4 } \\
\text { testing }\end{array}$} & \multicolumn{2}{|c|}{$\begin{array}{l}\text { Rate of mortality } \\
\text { at CD4 }\end{array}$} & \multirow{2}{*}{$\begin{array}{c}\text { Ratio of } \\
200-349 \\
\text { to }<200 \\
\text { mortality }\end{array}$} \\
\hline & & & $\begin{array}{c}200-349 \\
\left(\theta \lambda_{4}\right)\end{array}$ & $\begin{array}{l}<200 \\
\left(\lambda_{4}\right)\end{array}$ & \\
\hline Badri et $a l^{18}$ & Cape Town & $\begin{array}{l}\text { At least } \\
\text { 6-monthly }\end{array}$ & 0.08 & 0.27 & 0.30 \\
\hline Hargrove et $a l^{19}$ & $\begin{array}{l}\text { Zimbabwean women } \\
\text { followed postpartum }\end{array}$ & $\begin{array}{l}\text { Only at } \\
\text { baseline }\end{array}$ & $0.0293^{*}$ & 0.1442 & 0.20 \\
\hline Coutsoudis et $a l^{10}$ & $\begin{array}{l}\text { Women in KwaZulu-Natal } \\
\text { followed postpartum }\end{array}$ & $\begin{array}{l}\text { Only at } \\
\text { baseline }\end{array}$ & - & 0.1714 & - \\
\hline Mermin et $a l^{14}$ & $\begin{array}{l}\text { Ugandan patients, } \\
\text { receiving CTX }\end{array}$ & & - & 0.392 & - \\
\hline Van Oosterhout et $a l^{20}$ & Blantyre, Malawi & 4-monthly & 0.04 & 0.34 & 0.12 \\
\hline Seyler $e t a l^{21}$ & $\begin{array}{l}\text { Abidjan, Côte d'Ivoire } \\
\text { (patients receiving CTX) }\end{array}$ & 6-monthly & 0.077 & 0.316 & 0.24 \\
\hline eART-linc ${ }^{22}$ & $\begin{array}{l}\text { Patients from Uganda and } \\
\text { Côte d'Ivoire }\end{array}$ & & - & 0.192 & - \\
\hline Average & & & 0.06 & 0.26 & 0.22 \\
\hline
\end{tabular}

* Mortality rate in women with CD4 200-399. 
The probability of attrition in the first 6 months is calculated as $\kappa_{0}=k_{0} R$, and the annual probability of attrition after the first 6 months is calculated as $\kappa_{1}=k_{1} R$. In these equations, $k_{0}$ and $k_{1}$ represent the 'base rates', and $R$ is the ratio of the true attrition rates to the base rates. The base parameters $k_{0}$ and $k_{1}$ have been set at 0.105 and 0.065 respectively, based on data from the Western Cape public sector programme. ${ }^{23}$ A gamma prior distribution is specified for parameter $R$, to represent the uncertainty regarding the true attrition rates. This prior distribution has a mean of 1 , so that the prior means on parameters $\kappa_{0}$ and $\kappa_{1}$ are the same as the values assumed previously. ${ }^{24}$ In order to set the variance of the prior distribution, it is necessary to examine the variability in attrition rates between settings. Table 3 compares cumulative rates of attrition by 12 months after starting ART in various South African programmes. Rates of attrition by 12 months reach as high as $25 \%$ and $28 \%$ in the studies of Ford et al and Rosen et al respectively, almost double the rates of attrition in the Western Cape as a whole. However, it is possible that the rates of attrition observed in the Western Cape may overstate the true rates of attrition, since almost half of all Western Cape patients not retained in care by 12 months are considered 'lost to follow-up', and may therefore still be receiving ART elsewhere. Setting the standard deviation for the gamma prior on $R$ to 0.4 yields 2.5 and 97.5 percentiles of 0.38 to 1.92 respectively, which adequately reflects the extent of uncertainty around the ratio of the true attrition rate to that recorded in the Western Cape province.

Table 3: Cumulative attrition by 12 months after starting ART in South African patients

\begin{tabular}{|c|c|c|c|}
\hline Study & Location & $\mathrm{n}$ & Attrition \\
\hline Boulle et $a l^{23}$ & Whole of Western Cape & 12587 & $14.7 \%$ \\
\hline Bekker et $a l^{25}$ & Gugulethu, Western Cape & 1139 & $9 \%$ \\
\hline Coetzee et al $^{26}$ & Khayelitsha, Western Cape & 287 & $13.7 \%$ \\
\hline Fatti et al ${ }^{27}$ & $\begin{array}{l}\text { Sites in KwaZulu-Natal, Eastern Cape, } \\
\text { Western Cape, Mpumalanga }\end{array}$ & 29203 & $17.9 \%$ \\
\hline Ford et $a l^{28}$ & Lusikisiki, Eastern Cape & 1025 & $24.9 \%$ \\
\hline Mutevedzi et al ${ }^{29}$ & Umkhanyakude, KwaZulu-Natal & 5719 & $16.0 \%$ \\
\hline Rosen et $a l^{30}$ & Sites in Gauteng, Eastern Cape, Mpumalanga & 400 & $28.0 \%$ \\
\hline Vella et al $^{31}$ & Sites in KwaZulu-Natal & 2835 & $19 \%$ \\
\hline
\end{tabular}

It is important to note that in our model, the attrition rates $\kappa_{0}$ and $\kappa_{1}$ are defined as the rates at which patients permanently stop ART, either due to death or due to the patient not collecting their medication. Our model does not allow for increases in rates of attrition over time, although various South African studies have noted marked trends towards greater loss to follow-up over time. ${ }^{27,32,33}$ The reasons for not allowing for a trend towards increased attrition over time are:

- It has been acknowledged that some of the apparent increase in loss to follow-up may be due to increases in administrative error, ${ }^{32}$ i.e. because of the increasingly large volumes of patients, it is difficult to keep accurate records of when patients visit the clinics.

- Some of the apparent increase in loss to follow-up is also due to increasing levels of decentralization in the provision of ART, with patients not informing the clinic at 
which they started ART of their move to a nearer clinic. These patients who remain on ART despite being lost to follow-up are not included in our definition of attrition.

- Some of the apparent increase in loss to follow-up is also due to patients temporarily stopping ART; patients who started ART in the early years of the ART programme and resumed ART after interrupting therapy would not be considered lost to follow-up now, but patients who recently interrupted ART and have not yet resumed ART would be considered lost to follow-up in ART programme reporting. These patients who temporarily interrupt ART are not included in our definition of attrition.

- To the extent that there is a real increase in the rate at which patients stop taking their medication, it is likely to be partially offset by reductions in mortality rates. These reductions in mortality rates are likely to be the result of trends towards increasing baseline CD4 counts in patients starting ART over time. ${ }^{34}$ The net effect of rising treatment discontinuation and declining mortality may be relatively little change in overall rates of attrition over time.

(d) Proportion of ART attrition that is attributable to death

Although rates of loss to follow-up are often reported to be higher than rates of mortality in patients receiving ART, a recent review has shown that a substantial proportion of patients 'lost to follow-up' are actually dead, and many are in fact still receiving ART in other treatment centres ${ }^{35}$ (the latter are not included in our definition of 'attrition'). In this review, the average proportion of patients lost to follow-up after ART initiation who were found to have died when subsequently traced was $46 \%$ in African studies (range 27-87\%). Unpublished data from the IeDEA Southern Africa Collaboration suggest that after correcting for under-reporting of deaths, mortality rates after ART initiation are approximately $75 \%$ of the average ART attrition rates assumed in section (c). The $75 \%$ proportion may be an underestimate of the true mortality fraction, since the IeDEA Collaboration consists mostly of relatively well-resourced ART programmes from urban areas, which are not likely to be representative of the ART programme at a national level, and which may therefore have relatively low mortality. However, $75 \%$ could also be an over-estimate of the true mortality fraction, since the model parameter $v$ is defined to exclude individuals who died after they stopped taking ART, and the mortality rate estimated from the IeDEA data does not separate out 'deaths on ART' from 'deaths off ART'. The prior distribution assigned to the $v$ parameter is therefore a beta distribution with a mean of 0.75 and standard deviation of 0.10 (the 2.5 and 97.5 percentiles of this distribution are 0.53 and 0.92 respectively).

(e) Bias in reported ART programme statistics

It is quite likely that there is some degree of bias in reported ART programme statistics, though it is not immediately obvious whether the bias would be towards overstatement or understatement of patient totals. This section discusses the types of reporting error that are likely to emerge and the extent to which they may bias the reported patient totals. We consider separately two types of reported ART programme statistics: reported numbers of patients cumulatively enrolled and reported numbers of patients currently receiving ART.

\section{Reported numbers of patients cumulatively enrolled}

From the cumulative patient totals, it is possible to calculate $\bar{S}(t)$, the reported number of patients starting ART in year $t$, by subtracting the cumulative total at time $t$ from the 
cumulative total at time $t+1$. The true number of patients starting ART between time $t$ and time $t+1, S(t)$, is assumed to be some multiple of the reported number. Mathematically, $S(t)=\bar{S}(t)\left(1+b_{0} Z(t)\right)$, where $b_{0} Z(t)$ is an adjustment factor to allow for bias in the reported data. The $b_{0} Z(t)$ parameters are unknown, and are therefore included in the Bayesian analysis. Prior distributions are specified for the $b_{0} Z(t)$ factor in each year, and each prior is assumed to be a normal distribution with a mean of 0 (i.e. assuming that on average the reported totals are correct) and standard deviation of $b_{0}$, so that $Z(t)$ is a standard normal variate. The standard deviation, $b_{0}$, is set by noting that there are two potential sources of bias in reporting cumulative numbers of patients enrolled on ART.

The first source of bias is double-counting. This may occur because patients who move from one clinic to another get counted as having started ART twice (once at the first clinic and once at the second clinic). In a worst-case scenario, if all patients moving between clinics are double-counted, the ratio of the number of patients incorrectly counted as starting ART in year $t$ to the true number of patients starting ART in year $t$ is

$$
\frac{0.5(N(t)+N(t+1)) \alpha \chi}{S(t)}
$$

where $N(t)$ is the total number of patients on ART at time $t, \alpha$ is the annual rate at which patients are lost to follow-up and $\chi$ is the proportion of patients lost to follow-up who move to a different clinic. (Note that $\alpha \chi$ does not include the patients who are known to transfer out, as these patients would typically receive a referral letter informing their new treatment provider of their previous receipt of ART, so that they would not be double-counted.)

Estimates from previous analyses ${ }^{24}$ suggest that the ratio $0.5(N(t)+N(t+1)) / S(t)$ is typically between 1 and 2. Annual rates of loss to follow-up in South African ART programmes are typically between $5 \%$ and $15 \%$, though varying according to the maturity of the programme. ${ }^{32}$ Two separate studies in Johannesburg have found the proportion of patients lost to follow-up who initiate ART elsewhere to be $11.4 \%$ and $25.4 \% .^{36,37}$ If it is assumed that $\chi$ is unlikely to exceed 0.3 , then the ratio of the over-count to the true number of patients starting ART is unlikely to exceed $2 \times 0.15 \times 0.3=0.075$.

The second potential source of bias is late reporting of patients who have started ART. If the number of patients cumulatively enrolled on ART is correctly reported at the start of the year, but there is an average delay of one month in the reporting of new patients, by the end of the year, one might expect that the estimated number of new patients $\bar{S}(t)$ to under-state the true number by one twelfth (0.083), if it is assumed that patients were enrolled uniformly over the course of the year. Conversely, if the cumulative number on ART at the end of the year is correctly reported, but the number at the start of the year is subject to late reporting, the estimated number of new patients $\bar{S}(t)$ will over-state the true number. However, because numbers of patients enrolled are generally increasing over time, one would generally expect the bias due to late reporting to be a downward bias rather than an upward bias.

The net effect of any over-statement due to double-counting and any under-statement due to late reporting is likely to be relatively small, and we consider it unlikely that the factor $b_{0} Z(t)$ 
would be either less than -0.12 or more than 0.12 . The standard deviation $b_{0}$ parameter is therefore set to 0.06 , so that the prior $95 \%$ confidence interval on $b_{0} Z(t)$ is from -0.12 to 0.12 .

\section{Reported numbers of patients currently receiving ART}

Some provinces have recently switched to reporting the numbers of patients currently on ART rather than the numbers of patients cumulatively enrolled on ART - this has also been the standard reporting practice in the private and NGO sectors for several years. In this situation, the true number of patients currently on ART (or temporarily interrupted) is assumed to be some multiple of the reported number of patients on ART at time $t, \bar{N}(t)$. Mathematically, $N(t)=\bar{N}(t)\left(1+b_{1} Z(t)\right)$, where $b_{1} Z(t)$ is an adjustment factor to allow for bias in the reported data. As before, prior distributions are specified for the $b_{1} Z(t)$ factor in each year, and each prior is assumed to be a normal distribution with a mean of 0 and standard deviation $b_{1}$.

As in the estimation of $S(t)$, late reporting could lead to under-estimation of $N(t)$. If there is an average delay of 1 month in the reporting of patients who have started ART, then the extent of the under-estimation would be roughly $(1 / 12) \times 0.5(S(t-1)+S(t)) / N(t)$ at time $t$. Estimates from previous analyses ${ }^{24}$ suggest that the ratio $0.5(S(t-1)+S(t)) / N(t)$ is typically around 0.8 , so that a 1 -month reporting delay would correspond to a bias of roughly $0.8 / 12=$ $6.7 \%$.

An important source of bias in the context of cross-sectional data relates to the inclusion and exclusion of individuals who have stopped ART. Patients who have temporarily interrupted ART are included in our modelled number of patients on ART, $N(t)$, but might not be included in the reported number of patients on ART, $\bar{N}(t)$. However, patients who have permanently discontinued ART are excluded from $N(t)$ but might be included in $\bar{N}(t)$. In order to determine the net extent of the bias due to the inclusion/exclusion of treatment interrupters, it is necessary to make the following assumptions:

- Firstly, it is assumed that patients temporarily interrupt ART at a monthly rate of $\beta$. This is distinct from permanent ART interruption. Kranzer et $a l^{38}$ found that in Masiphumelele, the annual rate at which patients discontinue ART is 12.8 per 100 person years, and that $33 \%$ of these individuals ultimately resume ART. This suggests a value for $\beta$ of $0.128 \times 0.33 / 12=0.0035$.

- Secondly, it is assumed that patients permanently discontinue ART, or transfer to ART at another centre without informing their original treatment centre (so-called 'silent transfer'), at a monthly rate of $\gamma$. Based on the same data summarized in the previous point, this parameter might be estimated as $0.128 \times(1-0.33) / 12=0.0071$.

- Thirdly, it is assumed that when reporting numbers of patients currently on ART, programmes count patients as being on ART if they are not more than $w$ months late for a scheduled visit. In the Western Cape, patients are included in the total number of patients on ART if they are not more than 3 months late for their most recent scheduled visit, i.e. $w=3 .{ }^{39}$ Other provinces have been instructed to use the same definition in reporting numbers of patients currently on ART, but it is not clear to what extent this recommendation is being followed (Meg Osler, personal communication). In other ART programmes in developing countries, definitions have varied between 1 and 6 months late for a scheduled appointment. ${ }^{40}$ 
- Fourthly, it is assumed that patients who resume ART after interrupting therapy do so after an average of 7 months, based on the median time to ART resumption observed in the community of Masiphumelele. ${ }^{38}$ In a review of other studies that have estimated duration of treatment interruptions, the median delay was found to be 150 days. $^{41}$

Based on these assumptions, patients who permanently discontinue ART are included in $\bar{N}(t)$ but excluded from $N(t)$ for an average of $w$ months, and patients who temporarily discontinue ART are included in $N(t)$ but excluded from $\bar{N}(t)$ for an average of $(7-w)$ months. (By including in the definition of $\gamma$ those individuals who have silently transferred, we are also assuming that individuals who silently transfer are double-counted in $\bar{N}(t)$ for an average of $w$ months.) The percentage by which $\bar{N}(t)$ exceeds $N(t)$ is approximately

$$
\gamma w-\beta(7-w)
$$

which is $0.7 \%$ when $w=3$ months. For values of $w$ between 0 and 6 months, the percentage would be between $-2.5 \%$ and $3.9 \%$.

A third potential source of bias is replacement of current totals with cumulative totals in sites that lack the capacity to update their records of numbers of patients currently on ART. This may occur even when the overall policy in the province is to report numbers currently on ART. There is anecdotal evidence of this occurring in the Western Cape, where the overall policy has always been to report numbers of patients currently on ART, but since 2007 lack of technical support has led to certain clinics calculating the numbers of patients 'currently' on ART by simply adding the number of patients starting ART in the current period to the number reported as having been on ART in the previous period, without making any allowance for patients who have stopped ART (Meg Osler, personal communication). This would lead to some over-estimation of the numbers of patients currently on ART, but it is difficult to quantify the likely extent of this over-estimation.

In summary, there are three sources of bias to be considered when estimating $N(t)$ from $\bar{N}(t)$ : bias due to late reporting (bias as low as $-6.7 \%$ if there is a one-month average delay), bias due to inclusion/exclusion of ART interrupters and double counting of transfers (bias between $-2.5 \%$ and $4 \%$ ) and bias due to reporting of cumulative totals in some clinics (bias $>0$ ). We assume that the net bias, adding all three sources of error together, would be unlikely to be more than $8 \%$ (absolute) of the true total, and have therefore set the standard deviation $b_{1}$ to be 0.04 , so that the 2.5 and 97.5 percentiles of $b_{1} Z(t)$ are $-8 \%$ and $8 \%$ respectively.

\section{Limitations}

It is important to note that the $Z(t)$ factor is applied to all data sources in year $t$, both numbers newly enrolled and current totals (although with different standard deviations). It could be argued that it would be more realistic to allow different values of $Z(t)$ for different data sources reported in the same year, since different provinces and different providers are likely to have different reporting systems. By using the same value of $Z(t)$ for all data sources in year $t$, we are being deliberately conservative, allowing for more uncertainty in the accuracy of the reported ART statistics than would actually exist if the biases in the totals reported by different provinces/providers were independent of one another. However, it is debatable 
whether biases are truly independent, and assuming independence could lead to some underestimation of the extent of the uncertainty in the ART programme statistics.

It is also important to note that this approach does not make assumptions about correlation between $Z(t)$ and $Z(t+1)$. Positive correlation might be expected when dealing with reported numbers of patients currently on ART, since the same sources of bias are likely to persist from one year to the next. However, negative correlation might be expected when dealing with reported numbers of patients cumulatively enrolled. For example, if the cumulative patient total is exaggerated at the start of year $t$, but the cumulative totals reported at the start of years $t-1$ and $t+1$ are correct, then the number of patients starting ART in year $t-1$ will be overstated, but the number of patients starting ART in year $t$ will be understated. Because of the uncertainty about whether correlation is positive or negative, zero correlation has been assumed.

\section{(f) Priors on model outputs}

Although there is considerable uncertainty regarding the HIV parameters referred to in the previous sections, it is clear that any combination of model parameters that leads to an estimate of ART need smaller than the estimated number of patients starting ART in South Africa cannot be plausible. We therefore specify priors on the model outputs to prevent these implausible parameter combinations from being included in the posterior sample. The model output of interest is $r_{g}(t)$, the annual rate at which ART is initiated in year $t$, in ART-eligible individuals of sex $g$. The inverse of this rate is the average time from becoming eligible for ART to starting ART (in years). Individuals receiving pre-ART care should receive CD4 testing every 6 months, so that individuals in pre-ART care would be determined to be ARTeligible on average 3 months after their CD4 count drops below the ART-eligible threshold. If individuals are newly-diagnosed and determined to be ART-eligible, there is also likely to be a delay before they start ART. Ingle $e t a l^{42}$ found that in the Free State ART programme, the median time from enrolment into the ART programme to starting ART was 95 days, though this median dropped from 122 days in 2004 to 78 days in 2007. Some of this delay was due to a 3-week drug readiness training programme that all patients had to complete, and some of the delay was due to patients being referred to clinics where they could start ART. In a Cape Town study, ${ }^{43}$ the median time between enrolment into the ART programme and ART initiation was 34 days, although this did not include the time taken to assess ART eligibility. In a Durban study, ${ }^{44}$ the average time from when the CD4 test was performed (to assess eligibility) to when ART training began was 3.6 months. In another Cape Town study, ${ }^{45}$ $69.2 \%$ of patients who were diagnosed HIV-positive and had CD4 counts <200/ $\mu 1$ started ART within 2 months of HIV diagnosis. Using point-of-care CD4 testing, Faal et $a l^{46}$ found that $65 \%$ of patients in Johannesburg who were newly diagnosed and ART-eligible started ART within 3 months. However, because point-of-care CD4 testing is not generally used in South Africa, and delays in the collection of CD4 testing are usually significant, this is probably an over-estimate of the proportion of patients who would normally start ART within 3 months of diagnosis. Based on the reviewed evidence, it is considered unlikely that the average time from when individuals become ART-eligible to when they start ART would be less than 2 months (corresponding to $r_{g}(t)=6$ ). The prior distribution for $r_{g}(t)$ is therefore set to be uniform on the interval $[0,6]$, for all values of $t$ and $g$. This means that any parameter combination that leads to a modelled rate of ART initiation $>6$ per annum (for males or females in any year) will automatically be rejected. 


\section{Likelihood function}

The likelihood function measures how well the model fits the data, for a given set of model input parameters. In this analysis, two sources of data are included in the definition of the likelihood: CD4 data from surveys of HIV-positive adults, and age-specific HIV prevalence data from household surveys. A likelihood function is defined separately for each data source, and the total likelihood is the product of likelihood functions calculated for each data source.

(a) Likelihood function for CD4 data

Three different South African studies have measured the proportions of HIV-infected adults in different CD4 categories. The results of these three studies are summarized in Table 4. All three studies were conducted when access to antiretroviral treatment in South Africa was fairly limited, and the proportions can therefore be assumed to be representative of untreated individuals.

Table 4: Survey estimates of proportions of HIV-infected adults in different CD4 stages

\begin{tabular}{|c|c|c|c|c|c|c|c|}
\hline \multirow[b]{2}{*}{ Study } & \multirow{2}{*}{$\begin{array}{c}\text { Year } \\
\text { of } \\
\text { survey }\end{array}$} & \multirow[b]{2}{*}{$\begin{array}{l}\text { Population } \\
\text { sampled }\end{array}$} & \multirow{2}{*}{$\begin{array}{c}\# \\
\text { HIV+ } \\
\text { adults }\end{array}$} & \multicolumn{4}{|c|}{$\%$ with CD4 of } \\
\hline & & & & $>500$ & $\begin{array}{c}350- \\
500\end{array}$ & $\begin{array}{c}200- \\
349\end{array}$ & $<200$ \\
\hline Auvert et al ${ }^{9}$ & 2002 & $\begin{array}{l}\text { Households in } \\
\text { Orange Farm }\end{array}$ & 196 & 46.0 & 25.6 & 18.9 & 9.5 \\
\hline Rehle and Shisana ${ }^{47}$ & 2004 & Teachers & 444 & 27.9 & 19.8 & 30.0 & 22.3 \\
\hline Connelly et $a l^{48}$ & 2005 & Health workers & 74 & 35.1 & 17.6 & 28.4 & 18.9 \\
\hline
\end{tabular}

Suppose that if a set of parameters, represented by vector $\boldsymbol{\psi}$, is entered into the model, the model estimates that the proportions of ART-naïve individuals in stage $i$ of HIV infection, in year $t_{j}$, is $\pi_{i}\left(t_{j}, \boldsymbol{\psi}\right)$ (there are four possible stages of infection corresponding to the four untreated CD4 stages in Figure 1). Further suppose that the observed numbers of infected individuals in the $j^{\text {th }}$ study, conducted in year $t_{j}$, who are in stage $i$ is $n_{i j}$. It can then be assumed that the $n_{i j}$ terms are multinomially distributed, so that the likelihood function in respect of the $j^{\text {th }}$ study is equal to

$$
\left(\begin{array}{c}
n_{j} \\
n_{1 j} n_{2 j} n_{3 j} n_{4 j}
\end{array}\right) \pi_{1}\left(t_{j}, \boldsymbol{\psi}\right)^{n_{1 j}} \pi_{2}\left(t_{j}, \boldsymbol{\psi}\right)^{n_{2 j}} \pi_{3}\left(t_{j}, \boldsymbol{\psi}\right)^{n_{3 j}} \pi_{4}\left(t_{j}, \boldsymbol{\psi}\right)^{n_{4 j}},
$$

where $n_{j}$ is the total number of HIV-positive adults in the $j^{\text {th }}$ study. This is a fixed effects model, i.e. it is assumed that the CD4 distribution is the same for all South African populations that we might choose to sample. The assumption of a fixed effects framework is probably unrealistic, since some populations may be genetically different from others, some populations may be experiencing more advanced epidemics than others, etc. To account for variation in proportions between sub-populations, we define $\rho_{i j}$ as the true proportion of infected individuals in stage $i$, in the $j^{\text {th }}$ sub-population, and assume that the $\rho_{i j}$ terms are Dirichlet-distributed, i.e. 


$$
p\left(\boldsymbol{\rho}_{j} \mid \boldsymbol{\pi}\left(t_{j}, \boldsymbol{\psi}\right), \varphi\right)=\Gamma(\varphi) \prod_{i=1}^{4}\left(\rho_{i j} \pi_{i}\left(t_{j}, \boldsymbol{\psi}\right)-1\right) / \Gamma\left(\varphi \pi_{i}\left(t_{j}, \boldsymbol{\psi}\right)\right)
$$

where $\boldsymbol{\rho}_{j}$ and $\boldsymbol{\pi}\left(t_{j}, \boldsymbol{\psi}\right)$ represent the vectors of $\rho_{i j}$ and $\pi_{i}\left(t_{j}, \boldsymbol{\psi}\right)$ values respectively. Note that from the properties of the Dirichlet distribution,

$$
\begin{aligned}
& \mathrm{E}\left[\rho_{i j}\right]=\pi_{i}\left(t_{j}, \boldsymbol{\psi}\right) \\
& \operatorname{Var}\left[\rho_{i j}\right]=\frac{\pi_{i}\left(t_{j}\right)\left(1-\pi_{i}\left(t_{j}, \boldsymbol{\psi}\right)\right)}{\varphi+1}
\end{aligned}
$$

so that the $\varphi$ variable controls the variance of the random effects, and hence the variability in CD4 proportions across studies. For the purpose of this analysis, the $\varphi$ parameter is fixed at 188.5, the maximum likelihood estimate obtained in a previous analysis of the same CD4 data. ${ }^{49}$ The likelihood function in respect of study $j$ is then

$$
p\left(\mathbf{n}_{j} \mid \boldsymbol{\pi}\left(t_{j}, \boldsymbol{\psi}\right), \varphi\right)=\int_{\boldsymbol{\rho}_{j}} p\left(\mathbf{n}_{j} \mid \boldsymbol{\rho}_{j}\right) p\left(\boldsymbol{\rho}_{j} \mid \boldsymbol{\pi}\left(t_{j}, \boldsymbol{\psi}\right), \varphi\right) d \boldsymbol{\rho}_{j}
$$

where $\mathbf{n}_{j}$ represents the vector of $n_{i j}$ values. This likelihood can be more fully expressed as

$$
\int_{\rho_{1 j}} \int_{\rho_{2 j}} \int_{\rho_{3 j}} \int_{\rho_{4 j}} \Gamma(\varphi)\left(\begin{array}{c}
n_{j} \\
n_{1 j} n_{2 j} n_{3 j} n_{4 j}
\end{array}\right) \prod_{i=1}^{4} \frac{\rho_{i j}^{n_{i j}+\varphi \pi_{i}\left(t_{j}, \psi\right)-1}}{\Gamma\left(\varphi \pi_{i}\left(t_{j}, \psi\right)\right)} d \rho_{4 j} d \rho_{3 j} d \rho_{2 j} d \rho_{1 j}
$$

Note that after factoring out the terms that are independent of $\rho_{i j}$ in the above equation, the integral is itself of a Dirichlet form, and therefore integrates to 1 with the multiplication of an appropriate constant term. Hence

$$
p\left(\mathbf{n}_{j} \mid \boldsymbol{\pi}\left(t_{j}, \boldsymbol{\psi}\right), \varphi\right)=\frac{\Gamma(\varphi)}{\Gamma\left(n_{j}+\varphi\right)}\left(\begin{array}{c}
n_{j} \\
n_{1 j} n_{2 j} n_{3 j} n_{4 j}
\end{array}\right) \prod_{i=1}^{4} \frac{\Gamma\left(n_{i j}+\varphi \pi_{i}\left(t_{j}, \boldsymbol{\psi}\right)\right)}{\Gamma\left(\varphi \pi_{i}\left(t_{j}, \boldsymbol{\psi}\right)\right)} .
$$

For the purpose of simulating the posterior distribution, it is sufficient to calculate the log of the likelihood function and to exclude those terms that are independent of the parameters that we are trying to estimate (i.e. terms independent of $\boldsymbol{\psi}$ ). The total log likelihood is obtained by summing the values of the log likelihood for each individual study:

$$
l_{C}(\mathbf{n} \mid \boldsymbol{\psi})=\sum_{j=1}^{3} \sum_{i=1}^{4} \ln \left(\Gamma\left(n_{i j}+\varphi \pi_{i}\left(t_{j}, \boldsymbol{\psi}\right)\right)\right)-\ln \left(\Gamma\left(\varphi \pi_{i}\left(t_{j}, \boldsymbol{\psi}\right)\right)\right),
$$

where $\mathbf{n}$ is the matrix of $n_{i j}$ values.

(b) Likelihood function for HIV prevalence data

This component of the likelihood function is defined with reference to the HIV prevalence levels measured, by age and sex, in the national household surveys conducted by the Human 
Sciences Research Council (HSRC) in $2005^{50}$ and 2008. ${ }^{51}$ Suppose that $H_{g, x, t}(\boldsymbol{\psi})$ is the model estimate of HIV prevalence in individuals of sex $g$, aged $x$ to $x+4$ in year $t$, where the vector $\boldsymbol{\psi}$ represents the values of the model input parameters. The corresponding prevalence of HIV actually measured in the HSRC survey is represented by $y_{g, x, t}$. It is assumed that if $\boldsymbol{\psi}$ is the true set of parameter values, then the difference between the logit-transformed model estimate and the logit-transformed observed prevalence is normally distributed with zero mean, i.e.

$$
\log \left(\frac{y_{g, x, t}}{1-y_{g, x, t}}\right)=\log \left(\frac{H_{g, x, t}(\boldsymbol{\psi})}{1-H_{g, x, t}(\boldsymbol{\psi})}\right)+\varepsilon_{g, x, t},
$$

where $\varepsilon_{g, x, t} \sim N\left(0, \sigma_{g, x, t}^{2}\right)$, representing the random sampling error in the survey. The logit transformations ensure that the error terms are closer to normality and that the model error terms are roughly independent of the level of HIV prevalence. The $\sigma_{g, x, t}^{2}$ values are estimated from the $95 \%$ confidence intervals that have been published for the various survey estimates. The likelihood in respect of a single measurement is then calculated as

$$
p\left(y_{g, x, t} \mid \boldsymbol{\psi}\right)=\left(2 \pi \sigma_{g, x, t}^{2}\right)^{-0.5} \exp \left[-\frac{\left(\operatorname{logit}\left(y_{g, x, t}\right)-\operatorname{logit}\left(H_{g, x, t}(\psi)\right)\right)^{2}}{2 \sigma_{g, x, t}^{2}}\right]
$$

As before, it is sufficient to calculate the log of the likelihood and to exclude those terms that are independent of $\boldsymbol{\psi}$. The total $\log$ likelihood is obtained by summing the values of the $\log$ likelihood for each prevalence measurement:

$$
l_{H}(\mathbf{y} \mid \boldsymbol{\psi})=-\sum_{g} \sum_{x} \sum_{t} \frac{\left(\operatorname{logit}\left(y_{g, x, t}\right)-\operatorname{logit}\left(H_{g, x, t}(\boldsymbol{\psi})\right)\right)^{2}}{2 \sigma_{g, x, t}^{2}}
$$

where $\mathbf{y}$ represents the matrix of $y_{g, x, t}$ values. In this analysis, only HIV prevalence measurements in the 15-49 age range are used in calculating the likelihood, so that the summation in relation to $x$ is for age groups $15-19,20-24, \ldots, 45-49$.

Finally, the log likelihood in respect of the CD4 data, $l_{C}(\mathbf{n} \mid \psi)$, is added to the log likelihood in respect of the HIV prevalence data, $l_{H}(\mathbf{y} \mid \psi)$, to obtain the log of the total likelihood.

\section{Posterior simulation}

The posterior distribution represents the synthesis of the prior distributions assigned to the different parameters and the likelihood values calculated for each parameter combination. The likelihood function is simulated numerically, using Sampling Importance Resampling (SIR) ${ }^{52,53}$ This is a three-step procedure. In the first step of the algorithm, 10000 parameter combinations are randomly sampled from the prior distributions described in sections 2(a)-(e) (suppose that $\boldsymbol{\psi}_{i}$ represents the $i^{\text {th }}$ parameter combination that is sampled). In the second step of the algorithm, the log likelihood is calculated for each parameter combination $\left(l_{C}\left(\mathbf{n} \mid \boldsymbol{\psi}_{i}\right)+\right.$ 
$l_{H}\left(\mathbf{y} \mid \boldsymbol{\psi}_{i}\right)$ ), and an importance weight is assigned to each parameter combination based on this $\log$ likelihood. For the $i^{\text {th }}$ parameter combination, this weight is calculated as

$$
W_{i}= \begin{cases}\exp \left(l_{C}\left(\mathbf{n} \mid \boldsymbol{\psi}_{i}\right)+l_{H}\left(\mathbf{y} \mid \boldsymbol{\psi}_{i}\right)-\sum_{j \in A}\left\{l_{C}\left(\mathbf{n} \mid \boldsymbol{\psi}_{j}\right)+l_{H}\left(\mathbf{y} \mid \boldsymbol{\psi}_{j}\right)\right\}\right) & i \in A \\ 0 & i \notin A\end{cases}
$$

where $A$ is the set of model parameter combinations that yield estimates of $r_{g}(t)<6$ for all $g$ and $t$. Setting $W_{i}=0$ if $i \notin A$ means applying the prior distribution described in section 2(f) to the model outputs. In this respect our approach differs from the standard Bayesian approach. Bayesian melding generalizes the standard Bayesian approach by allowing for prior distributions to be assigned to model outputs, and not only to model inputs. ${ }^{54,55}$

In the final step of the SIR algorithm, a 'resample' of 1000 parameter combinations is drawn from the original sample, using the $\left\{W_{i}\right\}$ values as sample weights (sampling is with replacement). This sample constitutes a random sample from the posterior distribution. The model is then run for each parameter combination in this posterior sample in order to obtain an approximation to the distribution of model outputs (this distribution is summarized in terms of its mean and $95 \%$ confidence interval, for each model output).

In this analysis, the posterior sample of 1000 parameter combinations included 410 unique parameter combinations, and the most frequently sampled parameter combination accounted for only 13 parameter combinations ( $1.3 \%$ of the total). This indicates that the posterior sample is not unduly influenced by single parameter combinations that have unusually high likelihood values.

Table 5 compares the prior and posterior distributions for each of the model parameters. Posterior estimates suggest a more rapid rate of progression to CD4 $<350 / \mu 1$ than assumed $a$ priori, based on rates of CD4 decline in developed countries. However, rates of mortality after CD4 drops below 350/ $\mu$ l are marginally lower than those that have been assumed a priori, based on data in other African countries. The posterior mean for the $R$ parameter is substantially lower than the prior mean (0.74 compared to 1.00$)$, which suggests that the true rate of attrition in South Africa is lower than has been observed in the Western Cape. This is probably because our definition of attrition excludes individuals who temporarily interrupt ART or transfer between ART services without informing the clinic at which they originally received ART (both groups are likely to comprise a substantial proportion of patients considered 'lost to follow-up' in the Western Cape ART programme). The relatively low rate of attrition estimated in this analysis explains why the estimates of total patient numbers (Table 2 of the main text) are slightly higher than those estimated previously. ${ }^{24}$ 
Table 5: Comparison of prior and posterior distributions

\begin{tabular}{|c|c|c|c|}
\hline Parameter (symbol) & & $\begin{array}{c}\text { Prior distribution } \\
\text { (mean, } 95 \% \mathrm{CI} \text { ) }\end{array}$ & $\begin{array}{l}\text { Posterior distribution } \\
\quad(\text { mean, } 95 \% \mathrm{CI})\end{array}$ \\
\hline \multicolumn{4}{|l|}{ Parameters for untreated adults } \\
\hline Annual rate of progression from CD4 $>500$ to $350-500$ & $\lambda_{1}$ & $0.30(0.19-0.43)$ & $0.34(0.28-0.39)$ \\
\hline Annual rate of progression from CD4 $350-500$ to $200-349$ & $\lambda_{2}$ & $0.40(0.26-0.58)$ & $0.48(0.40-0.58)$ \\
\hline Annual rate of progression from CD4 200-349 to $<200$ & $\lambda_{3}$ & $0.35(0.23-0.50)$ & $0.32(0.25-0.39)$ \\
\hline Annual rate of HIV mortality if CD4 $<200$ & $\lambda_{4}$ & $0.25(0.13-0.40)$ & $0.21(0.16-0.27)$ \\
\hline $\begin{array}{l}\text { Ratio of HIV mortality at CD4 200-349 to HIV mortality } \\
\text { at CD4 <200 }\end{array}$ & $\theta$ & $0.15(0.07-0.26)$ & $0.13(0.05-0.24)$ \\
\hline \multicolumn{4}{|l|}{ Parameters for treated adults } \\
\hline Relative rate of attrition (as multiple of WC estimate) & $R$ & $1.00(0.38-1.92)$ & $0.74(0.27-1.34)$ \\
\hline Proportion of permanent loss to care that is due to death & $v$ & $0.75(0.53-0.92)$ & $0.74(0.53-0.92)$ \\
\hline Bias factors & $Z(t)$ & & \\
\hline Year 2001 & & $0(-1.96$ to 1.96$)$ & $0.02(-2.04$ to 1.79$)$ \\
\hline Year 2002 & & $0(-1.96$ to 1.96$)$ & $0.09(-1.81$ to 2.16$)$ \\
\hline Year 2003 & & $0(-1.96$ to 1.96$)$ & $0.00(-1.90$ to 1.94$)$ \\
\hline Year 2004 & & $0(-1.96$ to 1.96$)$ & $-0.07(-2.32$ to 1.99$)$ \\
\hline Year 2005 & & $0(-1.96$ to 1.96$)$ & $0.06(-2.08$ to 1.99$)$ \\
\hline Year 2006 & & $0(-1.96$ to 1.96$)$ & $0.11(-1.90$ to 2.11$)$ \\
\hline Year 2007 & & $0(-1.96$ to 1.96$)$ & $0.05(-2.03$ to 2.22$)$ \\
\hline Year 2008 & & $0(-1.96$ to 1.96$)$ & $0.07(-2.15$ to 1.97$)$ \\
\hline Year 2009 & & $0(-1.96$ to 1.96$)$ & $-0.01(-1.85$ to 2.11$)$ \\
\hline Year 2010 & & $0(-1.96$ to 1.96$)$ & $-0.12(-1.96$ to 1.76$)$ \\
\hline Year 2011 & & $0(-1.96$ to 1.96$)$ & $-0.39(-2.41$ to 1.44$)$ \\
\hline
\end{tabular}

In general, average posterior estimates of bias in reported ART statistics are not substantially different from zero. However, in 2011 there appears to be a more substantial upward bias in the reported ART programme statistics (the average values of $b_{0} Z(2011)$ and $b_{1} Z(2011)$ are -0.023 and -0.016 respectively, suggesting true patient numbers are roughly $2 \%$ lower than those reported, on average). This may be because high values of $Z(2011)$ are likely to lead to implausibly high rates of ART initiation in the most recent year $\left(r_{g}(t)>6\right)$, and such parameter values would be excluded from the posterior sample. 


\section{References}

1. Actuarial Society of South Africa. ASSA2008 AIDS and Demographic Model; 2011. Available from: http://aids.actuarialsociety.org.za. Accessed 5 April 2011.

2. Golub JE, Pronyk P, Mohapi L, Thsabangu N, Moshabela M, Struthers H, et al. Isoniazid preventive therapy, HAART and tuberculosis risk in HIV-infected adults in South Africa: a prospective cohort. AIDS. 2009; 23(5):631-6.

3. Hanrahan CF, Golub JE, Mohapi L, Tshabangu N, Modisenyane T, Chaisson RE, et al. Body mass index and risk of tuberculosis and death. AIDS. 2010;24(10):1501-8.

4. Hussain A, Moodley D, Naidoo S, Esterhuizen TM. Pregnant women's access to PMTCT and ART services in South Africa and implications for universal antiretroviral treatment. PLoS One. 2011; 6(12):e27907.

5. Stinson K, Boulle A, Coetzee D, Abrams EJ, Myer L. Initiation of highly active antiretroviral therapy among pregnant women in Cape Town, South Africa. Trop Med Int Health. 2010; 15(7):825-32.

6. Horwood C, Haskins L, Vermaak K, Phakathi S, Subbaye R, Doherty T. Prevention of mother to child transmission of HIV (PMTCT) programme in KwaZulu-Natal, South Africa: an evaluation of PMTCT implementation and integration into routine maternal, child and women's health services. Trop Med Int Health. 2010; 15(9):992-9.

7. Médecins Sans Frontières. Khayelitsha Annual Activity Report, 2008-2009. Cape Town; 2010. Available from:

http://www.msf.org.za/Docs/Khayelitsha/Khayelitsha_Report_2008-2009.pdf. Accessed 24 Feb 2010.

8. van der Merwe K, Chersich MF, Technau K, Umurungi Y, Conradie F, Coovadia A. Integration of antiretroviral treatment within antenatal care in Gauteng Province, South Africa. J Acquir Immun Defic Syndr. 2006; 43(5):577-81.

9. Auvert B, Males S, Puren A, Taljaard D, Carael M, Williams B. Can highly active antiretroviral therapy reduce the spread of HIV? A study in a township of South Africa. $J$ Acquir Immun Defic Syndr. 2004; 36(1):613-21.

10. Coutsoudis A, England K, Rollins N, Coovadia H, Newell ML, Bland R. Women's morbidity and mortality in the first 2 years after delivery according to HIV status. AIDS. 2010; 24(18):2859-66.

11. Longini IM, Clark WS, Gardner LI, Brundage JF. The dynamics of CD4+ Tlymphocyte decline in HIV-infected individuals: a Markov modeling approach. $J$ Acquir Immun Defic Syndr. 1991; 4(11):1141-7.

12. Satten GA, Longini IM. Markov chains with measurement error: estimating the 'true' course of a marker of the progression of human immunodeficiency virus disease. Appl Statist. 1996; 45(3):275-309.

13. Hendriks JC, Craib KJ, Veugelers PJ, van Druten HA, Coutinho RA, Schechter MT, et al. Secular trends in the survival of HIV-infected homosexual men in Amsterdam and Vancouver estimated from a death-included CD4-staged Markov model. Int J Epidemiol. 2000; 29(3):565-72.

14. Mermin J, Were W, Ekwaru JP, Moore D, Downing R, Behumbiize P, et al. Mortality in HIV-infected Ugandan adults receiving antiretroviral treatment and survival of their HIVuninfected children: a prospective cohort study. Lancet. 2008; 371(9614):752-9.

15. Malone JL, Simms TE, Gray GC, Wagner KF, Burge JR, Burke DS. Sources of variability in repeated T-helper lymphocyte counts from human immunodeficiency virus type 1-infected patients: total lymphocyte count fluctuations and diurnal cycle are important. $J$ Acquir Immun Defic Syndr. 1990; 3(2):144-51. 
16. Ritchie AW, Oswald I, Micklem HS, Boyd JE, Elton RA, Jazwinska E, et al. Circadian variation of lymphocyte subpopulations: a study with monoclonal antibodies. BMJ. $1983 ; 286(6380): 1773-5$.

17. Miyawaki T, Taga K, Nagaoki T, Seki H, Suzuki Y, Taniguchi N. Circadian changes of T lymphocyte subsets in human peripheral blood. Clin Exp Immunol. 1984; 55(3):618-22.

18. Badri M, Lawn SD, Wood R. Short-term risk of AIDS or death in people infected with HIV-1 before antiretroviral therapy in South Africa: a longitudinal study. Lancet. 2006; 368(9543):1254-9.

19. Hargrove JW, Humphrey JH. Mortality among HIV-positive postpartum women with high CD4 cell counts in Zimbabwe. AIDS. 2010; 24(3):F11-4.

20. van Oosterhout JJ, Laufer MK, Graham SM, Thumba F, Perez MA, Chimbiya N, et al. A community-based study of the incidence of trimethoprim-sulfamethoxazole-preventable infections in Malawian adults living with HIV. J Acquir Immun Defic Syndr. 2005; 39(5):626-31.

21. Seyler C, Messou E, Gabillard D, Inwoley A, Alioum A, Anglaret X. Morbidity before and after HAART initiation in sub-Saharan African HIV-infected adults: a recurrent event analysis. AIDS Res Hum Retroviruses. 2007; 23(11):1338-47.

22. Eligibility for ART in lower income countries collaboration. Duration from seroconversion to eligibility for antiretroviral therapy and from ART eligibility to death in adult HIV-infected patients from low and middle-income countries: collaborative analysis of prospective studies. Sex Transm Infect. 2008; 84 (Suppl i):i31-6.

23. Boulle A, Bock P, Osler M, Cohen K, Channing L, Hilderbrand K, et al. Antiretroviral therapy and early mortality in South Africa. Bull WHO. 2008;86(9):678-87.

24. Adam MA, Johnson LF. Estimation of adult antiretroviral treatment coverage in South Africa. S Afr Med J. 2009; 99(9):661-7.

25. Bekker LG, Myer L, Orrell C, Lawn S, Wood R. Rapid scale-up of a communitybased HIV treatment service: programme performance over 3 consecutive years in Guguletu, South Africa. S Afr Med J. 2006; 96(4):315-20.

26. Coetzee D, Hilderbrand K, Boulle A, Maartens G, Louis F, Labatala V, et al. Outcomes after two years of providing antiretroviral treatment in Khayelitsha, South Africa. AIDS. 2004; 18:887-95.

27. Fatti G, Grimwood A, Bock P. Better antiretroviral therapy outcomes at primary healthcare facilities: an evaluation of three tiers of ART services in four South African provinces. PLoS One. 2010; 5(9):e12888.

28. Ford N, Reuter H, Bedelu M, Schneider H, Reuter H. Sustainability of long-term treatment in a rural district: the Lusikisiki model of decentralised HIV/AIDS care. South Afr J HIV Med. 2006; 7(4):17-22.

29. Mutevedzi PC, Lessells RJ, Heller T, Bärnighausen T, Cooke GS, Newell ML. Scaleup of a decentralized HIV treatment programme in rural KwaZulu-Natal, South Africa: does rapid expansion affect patient outcomes? Bull WHO. 2010; 88(8):593-600.

30. Rosen S, Long L, Sanne I. The outcomes and outpatient costs of different models of antiretroviral treatment delivery in South Africa. Trop Med Int Health. 2008; 13(8):1005-15.

31. Vella V, Govender T, Dlamini S, Taylor M, Moodley I, David V, et al. Retrospective study on the critical factors for retaining patients on antiretroviral therapy in KwaZulu-Natal, South Africa. J Acquir Immun Defic Syndr. 2010; 55(1):109-16.

32. Cornell M, Grimsrud A, Fairall L, Fox MP, van Cutsem G, Giddy J, et al. Temporal changes in programme outcomes among adult patients initiating antiretroviral therapy across South Africa, 2002-2007. AIDS. 2010; 24(14):2263-70. 
33. Nglazi MD, Lawn SD, Kaplan R, Kranzer K, Orrell C, Wood R, et al. Changes in programmatic outcomes during 7 years of scale-up at a community-based antiretroviral treatment service in South Africa. J Acquir Immun Defic Syndr. 2011; 56(1):e1-8.

34. Cornell M, Technau K, Fairall L, Wood R, Moultrie H, van Cutsem G, et al. Monitoring the South African antiretroviral programme 2003-2007: the IeDEA Southern Africa Collaboration. S Afr Med J. 2009; 99(9):653-60.

35. Brinkhof MW, Pujades-Rodriguez M, Egger M. Mortality of patients lost to follow-up in antiretroviral treatment programmes in resource-limited settings: systematic review and meta-analysis. PLoS One. 2009; 4(6):e5790.

36. Maskew M, MacPhail P, Menezes C, Rubel D. Lost to follow up: contributing factors and challenges in South African patients on antiretroviral therapy. S Afr Med J. 2007; 97(9):853-7.

37. Dalal RP, Macphail C, Mqhayi M, Wing J, Feldman C, Chersich MF, et al. Characteristics and outcomes of adult patients lost to follow-up at an antiretroviral treatment clinic in Johannesburg, South Africa. J Acquir Immun Defic Syndr. 2008; 47(1):101-7.

38. Kranzer K, Lewis JJ, Ford N, Zeinecker J, Orrell C, Lawn SD, et al. Treatment interruption in a primary care antiretroviral therapy program in South Africa: cohort analysis of trends and risk factors. J Acquir Immun Defic Syndr. 2010; 55(3):e17-23.

39. Western Cape Department of Health. Mapping Progress of the Western Cape Antiretroviral Programme, 2008/09. Cape Town: Provincial Government of the Western Cape; 2009.

40. Chi BH, Cantrell RA, Mwango A, Westfall AO, Mutale W, Limbada M, et al. An empirical approach to defining loss to follow-up among patients enrolled in antiretroviral treatment programs. Am J Epidemiol. 2010; 171(8):924-31.

41. Kranzer K, Ford N. Unstructured treatment interruption of antiretroviral therapy in clinical practice: a systematic review. Trop Med Int Health. 2011; 16(10):1297-1313.

42. Ingle SM, May M, Uebel K, Timmerman V, Kotze E, Bachmann M, et al. Outcomes in patients waiting for antiretroviral treatment in the Free State Province, South Africa: prospective linkage study. AIDS. 2010; 24(17):2717-25.

43. Lawn SD, Myer L, Harling G, Orrell C, Bekker LG, Wood R. Determinants of mortality and nondeath losses from an antiretroviral treatment service in South Africa: implications for program evaluation. Clin Infect Dis. 2006; 43(6):770-6.

44. Bassett IV, Wang B, Chetty S, Mazibuko M, Bearnot B, Giddy J, et al. Loss to care and death before antiretroviral therapy in Durban, South Africa. J Acquir Immun Defic Syndr. 2009; 51(2):135-9.

45. Govindasamy D, van Schaik N, Kranzer K, Wood R, Mathews C, Bekker LG. Linkage to HIV care from a mobile testing unit in South Africa by different CD4 count strata. J Acquir Immun Defic Syndr. 2011; 58(3):344-52.

46. Faal M, Naidoo N, Glencross DK, Venter WDF, Osih R. Providing immediate CD4 count results at HIV testing improves ART initiation. J Acquir Immun Defic Syndr. 2011; 58(3):e54-9.

47. Rehle TM, Shisana O. Estimates of eligibility for antiretroviral treatment (ART) and projected ART impact on AIDS mortality among South African educators. SAHARA J. 2005; 2(3):304-10.

48. Connelly D, Veriava Y, Roberts S, Tsotetsi J, Jordan A, DeSilva E, et al. Prevalence of HIV infection and median CD4 counts among health care workers in South Africa. S Afr Med J. 2007; 97(2):115-20. 
49. Johnson LF, Kranzer K, Middelkoop K, Wood R. A model of the impact of HIV/AIDS and antiretroviral treatment in the Masiphumelele community: Centre for Infectious Disease Epidemiology and Research; 2011. Available from:

http://webdav.uct.ac.za/depts/epi/publications/documents/Masiphumelele\%20ART\%20model \%202011.pdf. Accessed 29 Oct 2011.

50. Shisana O, Rehle T, Simbayi LC, Parker W, Zuma K, Bhana A, et al. South African National HIV Prevalence, HIV Incidence, Behaviours and Communication Survey, 2005. Cape Town: HSRC Press; 2005. Available from: http://www.hsrcpress.ac.za. Accessed 1 Dec 2005.

51. Shisana O, Rehle T, Simbayi LC, Zuma K, Jooste S, Pillay-van Wyk V, et al. South African national HIV prevalence, incidence, behaviour and communication survey, 2008: A turning tide among teenagers? Cape Town: Human Sciences Research Council; 2009. Available from: http://www.hsrcpress.ac.za. Accessed 9 June 2009.

52. Smith AFM, Gelfand AE. Bayesian statistics without tears - a sampling resampling perspective. Am Stat. 1992; 46(2):84-8.

53. Rubin DB. Using the SIR algorithm to simulate posterior distributions. In: Bernardo JM, DeGroot MH, Lindley DV, Smith AFM, editors. Bayesian Statistics 3. Oxford, U.K.: Oxford University Press; 1988. p. 395-402.

54. Poole D, Raftery AE. Inference for deterministic simulation models: The Bayesian melding approach. J Am Stat Assoc. 2000; 95(452):1244-55.

55. Alkema L, Raftery AE, Clark SJ. Probabilistic projections of HIV prevalence using Bayesian melding. Ann Appl Stat. 2007; 1(1):229-48. 